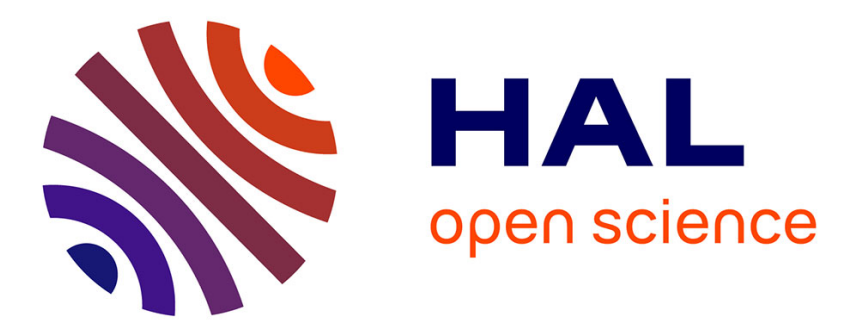

\title{
Synergistic role of nucleotides and lipids for the self-assembly of Shs1 septin oligomers
}

Cyntia Taveneau, Rémi Blanc, Gerard Pehau-Arnaudet, Aurélie Di Cicco, Aurélie Bertin

\section{- To cite this version:}

Cyntia Taveneau, Rémi Blanc, Gerard Pehau-Arnaudet, Aurélie Di Cicco, Aurélie Bertin. Synergistic role of nucleotides and lipids for the self-assembly of Shs1 septin oligomers. Biochemical Journal, 2020, 10.1042/BCJ20200199 . hal-02920169

\section{HAL Id: hal-02920169 \\ https://hal.science/hal-02920169}

Submitted on 24 Aug 2020

HAL is a multi-disciplinary open access archive for the deposit and dissemination of scientific research documents, whether they are published or not. The documents may come from teaching and research institutions in France or abroad, or from public or private research centers.
L'archive ouverte pluridisciplinaire HAL, est destinée au dépôt et à la diffusion de documents scientifiques de niveau recherche, publiés ou non, émanant des établissements d'enseignement et de recherche français ou étrangers, des laboratoires publics ou privés. 


\title{
Synergistic role of nucleotides and lipids for the self-assembly of Shs1 septin oligomers
}

\author{
Cyntia Taveneau $^{1,2, \star}$, Rémi Blanc ${ }^{3}$, Gérard Péhau-Arnaudet ${ }^{4}$, Aurélie Di Cicco ${ }^{1,2}$ and $\odot$ Aurélie Bertin $^{1,2}$

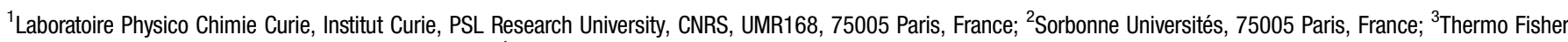 \\ Scientific, 3 Impasse Rudolf Diesel, Merignac, France; ${ }^{4}$ Ultrastructural Biolmaging UTechS UBI, UMR 3528, CNRS, Institut Pasteur, 75015 Paris, France
}

Correspondence: Aurélie Bertin (aurelie.bertin@curie.fr)

${ }^{*}$ Current address: Biomedicine Discovery Institute, Department of Biochemistry and Molecular Biology, Monash University, Clayton, Australia.

Received: 9 March 2020 Revised: 7 July 2020 Accepted: 9 July 2020

Accepted Manuscript online: 9 July 2020

Version of Record published: 29 July 2020
Budding yeast septins are essential for cell division and polarity. Septins assemble as palindromic linear octameric complexes. The function and ultra-structural organization of septins are finely governed by their molecular polymorphism. In particular, in budding yeast, the end subunit can stand either as Shs1 or Cdc11. We have dissected, here, for the first time, the behavior of the Shs1 protomer bound to membranes at nanometer resolution, in complex with the other septins. Using electron microscopy, we have shown that on membranes, Shs1 protomers self-assemble into rings, bundles, filaments or twodimensional gauzes. Using a set of specific mutants we have demonstrated a synergistic role of both nucleotides and lipids for the organization and oligomerization of budding yeast septins. Besides, cryo-electron tomography assays show that vesicles are deformed by the interaction between Shs1 oligomers and lipids. The Shs1-Shs1 interface is stabilized by the presence of phosphoinositides, allowing the visualization of micrometric long filaments formed by Shs1 protomers. In addition, molecular modeling experiments have revealed a potential molecular mechanism regarding the selectivity of septin subunits for phosphoinositide lipids.

\section{Introduction}

At the plasma membrane, in budding yeast Saccharomyces cerevisiae, septins assemble into a collarshaped structure at the bud neck during cell division (Figure 1A). The collar splits into two individual rings at the onset of cytokinesis after the remodeling of the ultrastructure of septins throughout cell division $[1,2]$. Five mitotic septins are expressed $[3,4]$ and self-assemble into rod-like conserved palindromic hexamers Cdc12-Cdc3-Cdc10-Cdc10-Cdc3-Cdc12 capped by either Cdc11 or Shs1 septin proteins at their ends [5-8] (Figure 1B). They possess a core guanine nucleotide-binding domain (GBD) flanked by N- and/or C-terminal extension (CTE) of various lengths $[9,10]$ (Figure 1B). They further self-assemble into non-polar filaments [5,7,11]. The assembly of Cdc11 capped complexes has been extensively analyzed both in solution and bound to membranes [12-14]. They form non-polar paired filaments or bundles [5] and sheets of filaments on PI(4,5)P2 doped biomimetic membranes [14] (Figure 1C, right). In solution and cell-free assays, Shs1 capped complexes interact laterally to oligomerize into bundled rings (Figure 1C, left), spirals and into gauzes [7] but are poorly able to associate end to end and thus to polymerize in solution [15]. In vivo, the GTPase activity of the Cdc12 subunit is responsible for the incorporation of either Cdc11 or Shs1 [16]. Shs1 interacts preferentially with $\mathrm{Cdc12}$ bound to GDP while $\mathrm{Cdcl1}$ is found in complex with non-hydrolyzed GTP-Cdc12 [16]. Hybrid protomers including both Cdc11 and Shs1 are not self-assembled [11]. Throughout the cell cycle, major reorganizations of septins are observed and are still not fully understood. The molecular versatility of septins, incorporating either Shs1 or Cdc11 likely finely tunes the remodeling of the septin architecture and affect the biophysical properties of the filaments [11]. It is essential to dissect, in vitro, the behavior of the Shs1 proto-filaments in a biomimetic environment. 
A

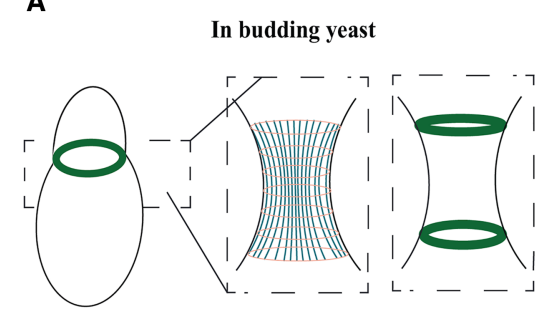

C

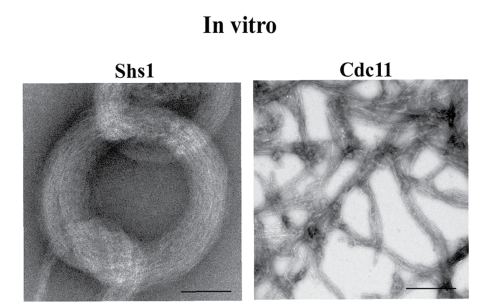

B

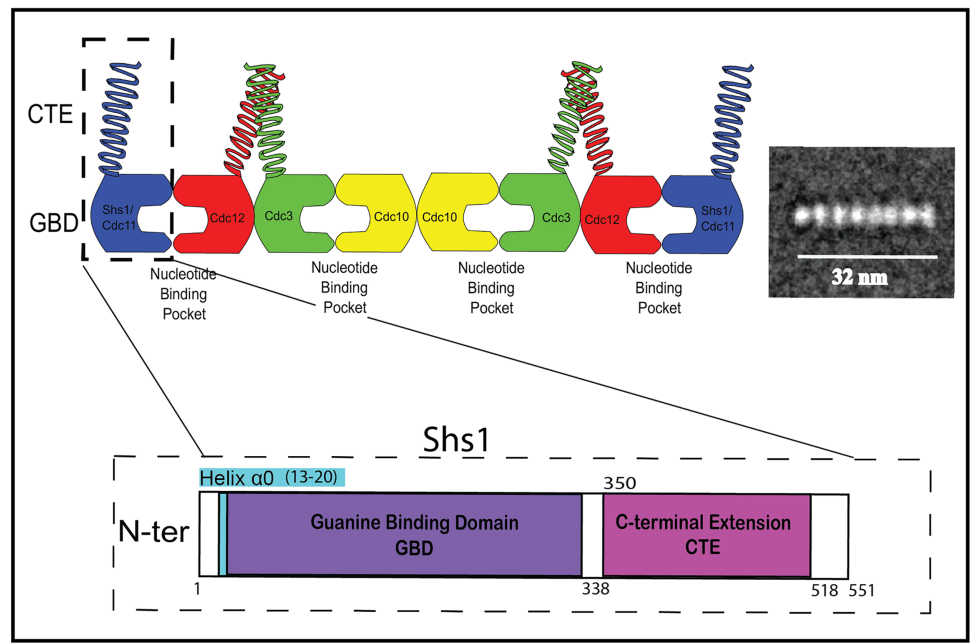

Figure 1. Organization of septin proteins.

(A) Mitotic septin organization during yeast division. Septins assemble into a highly dynamic platform at the collar bud neck, displaying an hourglass structure which is divided into two rings right before the separation of the daughter and mother cells (adapted from Vrabioiu et al. [56]). (B) Septin global architecture and Palindromic organization of mitotic septins in Shs1 oct. Septins are composed of two domains: a conserved and globular domain called Guanine binding domain (GBD) and a second domain, more variable, called C-terminal extension (CTE). Some septins (Cdc12, 3, 11 and Shs1) display a putative coiled-coil domain (CC) in their CTE. A density map of such octamers, obtained by electron microscopy and single-particle averaging, is displayed at the right of the panel. (C) Cdc11 and Shs1 capping subunits modulate the assembly of mitotic septins from S. cerevisiae. In solution, Shs1 capped octamers assemble into rings. Cdc11 capped protomers assemble into long filaments and bundle of filaments. Scale bar: $100 \mathrm{~nm}$.

Until now, in vitro studies describing the organization of the Shs1 capped octameric complex (designated as Shs1 $1_{\text {oct }}$ in the text) of septins were carried out mostly in solution and at a rather low resolution [7] even though, in vivo, septins interact with the inner plasma membrane. Recently, using TIRF microscopy, Shs1 complexes were shown to assemble at a micrometer scale, into curved structures on a lipid bilayer [11]. In this report, we provide additional and novel insights into the organization, at the nanometer scale, of the Shs1 capped complexes associated with solid flat or deformable biomimetic membranes doped with phosphoinositides. We have shown that Shs1 capped complexes, bound to membranes, display a high structural plasticity and sensitivity to nucleotides GTP and/or GDP in excess. Besides, we have characterized the interaction of septins with lipids from a molecular point of view, leading to new hypothesis regarding the specificity of L- $\alpha$-phosphatidylinositol-4,5-bisphosphate $\left(\mathrm{PI}(4,5) \mathrm{P}_{2}\right)$ with the $S$. cerevisiae mitotic septins.

\section{Methods}

\section{Reagents}

All lipids used in the following experiments were purchased from Avanti Polar Lipids: L- $\alpha$-phosphatidylinositol, PI (bovine liver, code 840042P); L- $\alpha$-phosphatidylinositol-4-sphosphate, PI(4)P (porcine brain; code 840045X), L- $\alpha$ phosphatidylinositol-4,5-bisphosphate, PI(4,5)P2 (18:1) (synthetic, code 850155); 1-stearoyl-2-arachidonoyl-snglycero-3-phospho-(1'-myo-inositol-3', $4^{\prime}, 5^{\prime}$-trisphosphate), PI(3,4,5)P3(synthetic, code 850166P); 1,2-dioleoyl-snglycero-3-phosphoethanolamine, DOPE (code 850725P); 1,2-dioleoyl-sn-glycero-3-phospho-L-serine, DOPS (code 840035P); 1,2-dioleoyl-sn-glycero-3-phosphocholine, DOPC (850375P).

\section{Septin expression and purification}

Septin complexes containing Shs1 (wild type or mutants) and Cdc11 were expressed in Escherichia coli BL21 (DE3) Star cells (ThermoFisher Scientific, ref C601003) as previously described in Bertin et al. [5] for Cdc11 
complexes; Garcia et al. [7] for Shs1 complexes with slight changes. Cdc12 was the only subunit to be expressed as a fusion protein with a hexa-histidines tag.

Shs1, Cdc3, His6-Cdc12 and Cdc10 were co-expressed in E. coli BL21(DE3)Star (ThermoFisher Scientific, ref C601003) from a pCod vector (Shs1), pMB vector (Cdc3) and pColADuet vector (Cdc10 and 12) (plasmid from Garcia et al. [7]). The cultures were grown to $\mathrm{A} 600 \mathrm{~nm}=1$ then cooled down to $17^{\circ} \mathrm{C}$ and induced with isopropyl- $\beta$-D-thiogalactoside (Euromedex, ref EU0008-A) to $1 \mathrm{mM}$ final overnight. Bacterial pellets were collected by centrifugation at $5000 \mathrm{~g}$ for $10 \mathrm{~min}$. They were flash frozen and stored at $-80^{\circ} \mathrm{C}$. A pellet from $1.5 \mathrm{l}$ of culture was re-suspended in $50 \mathrm{ml}$ of lysis buffer $\left(50 \mathrm{mM}\right.$ Tris- $\mathrm{HCl} \mathrm{pH8,} 300 \mathrm{mM} \mathrm{NaCl}, 5 \mathrm{mM} \mathrm{MgCl}_{2}, 40 \mu \mathrm{M}$ GDP, $0.5 \%$ (v/v) Tween 20, $12 \%$ glycerol, $5 \mathrm{mM} \beta$-mercaptoethanol, $10 \mathrm{mM}$ Imidazole). After thawing, one tablet of protease inhibitor mix (Complete Mini EDTA-free Protease Inhibitor Cocktail, Roche), about 1250 Units of benzonase (Sigma-Aldrich, ref E1014) and $0.5 \mathrm{mg} \mathrm{ml}^{-1}$ of lysozyme were added. After stirring for 30 $\min$ at $4^{\circ} \mathrm{C}$, the mixture was sonicated until the lysate was fluid and not viscous. The lysate was clarified at $4^{\circ} \mathrm{C}$ by centrifugation at $14000 \mathrm{~g}$ for $30 \mathrm{~min}$ and filtrated with a $0.22 \mu \mathrm{m}$ PES membrane filter. The resulting supernatant fraction was subjected to $\mathrm{Ni}^{2+}$-affinity chromatography (HisTrap FF $5 \mathrm{ml}$; GE Healthcare) after equilibration of the column into the wash buffer $(50 \mathrm{mM}$ Tris- $\mathrm{HCl}, \mathrm{pH} 8.0,300 \mathrm{mM} \mathrm{NaCl}, 20 \mathrm{mM}$ imidazole, $5 \mathrm{mM}$ $\mathrm{MgCl}_{2}, 0.1 \mathrm{mM}$ TCEP). After washing the column, the elution was performed (elution buffer: $50 \mathrm{mM}$ Tris$\mathrm{HCl}$, pH 8.0, $450 \mathrm{mM} \mathrm{NaCl}, 356 \mathrm{mM}$ imidazole, $5 \mathrm{mM} \mathrm{MgCl}_{2}, 0.1 \mathrm{mM}$ TCEP) and protein was collected into fractions of $1 \mathrm{ml}$. Size-exclusion chromatography (HiLoad 16/60 Superdex 200; GE Healthcare) was performed on the peak eluate fractions (buffer: $300 \mathrm{mM} \mathrm{NaCl}, 50 \mathrm{mM}$ Tris- $\mathrm{HCl}, \mathrm{pH} 8.0$, and $0.1 \mathrm{mM}$ TCEP). The peak size-exclusion eluate fractions were pooled, desalted with PD10 Sephadex G-25 columns (GE Healthcare) with a $50 \mathrm{mM}$ Tris- $\mathrm{HCl}$ pH8 buffer. Fractions containing the protein were transferred into an ion-exchange chromatography column (Resource Q $1 \mathrm{ml}$; GE Healthcare) and eluted with a gradient ranging from 0 to $500 \mathrm{mM}$ $\mathrm{NaCl}$ in $50 \mathrm{mM}$ Tris- $\mathrm{HCl} \mathrm{pH} 8.0$, and $0.1 \mathrm{mM}$ TCEP. Proteins were stored into small aliquots at $-80^{\circ} \mathrm{C}$ after flash freezing.

\section{Shs1 auto-assembly studies in solution}

Septins were diluted in $50 \mathrm{mM}$ Tris- $\mathrm{HCl} \mathrm{pH} 8,20 \mathrm{mM} \mathrm{NaCl}$ (plus $2 \mathrm{mM} \mathrm{MgCl}_{2}$ in some cases) (namely low salt conditions) to a final concentration of $0.01 \mathrm{mg} \mathrm{ml}^{-1}$. A $4 \mathrm{mM}$ of GTP Lithium salt (Roche; ref 11140957001) or GDP (Sigma-Aldrich; ref G7127) were added in specific assays. Samples were incubated from $10 \mathrm{~min}$ to $\sim 1 \mathrm{~h}$ at $4^{\circ} \mathrm{C}$ before being negatively stained. For high salt conditions, septins were diluted in $50 \mathrm{mM}$ Tris- $\mathrm{HCl} \mathrm{pH} 8$ (plus $2 \mathrm{mM} \mathrm{MgCl}_{2}$ in some cases) and $300 \mathrm{mM} \mathrm{NaCl}$.

\section{Lipid monolayer assays}

All of the assays were designed as duplicates or triplicates and the experiments were all repeated at least three times. The wells of a Teflon block were filled to capacity $(20 \mu \mathrm{l})$ with high salt $(50 \mathrm{mM}$ Tris- $\mathrm{HCl} \mathrm{pH} 8, \mathrm{NaCl}$ $300 \mathrm{mM}, \mathrm{MgCl}_{2} 2 \mathrm{mM}$ ) or low salt $\left(50 \mathrm{mM}\right.$ Tris- $\mathrm{HCl} \mathrm{pH} 8,10 \mathrm{mM} \mathrm{NaCl}, \mathrm{MgCl}_{2} 2 \mathrm{mM}$ ) solutions containing septin complexes. A drop $(\sim 0.5 \mu \mathrm{l})$ of lipid solution in chloroform was added to the surface of the solution. The lipid composition was chosen as follows: $80 \%$ DOPC, $10 \%$ PS and 10\% of variable negatively charged lipid (PS or Phosphoinositides). The Teflon block was inserted in a humidified Petri dish and equilibrated $5 \mathrm{~h}$ at $4^{\circ}$ C. To recover the lipid monolayer and absorbed protein, a grid coated with a continuous hydrophobic carbon film (Electron microscopy Sciences, ref CF300-CU) was positioned on the surface of the solution. The grids were then blotted and stained with a solution of $2 \%$ uranyl formate before being analyzed by EM. All of the experiments were repeated at least once. All of the experiments were carried out in the presence of $2 \mathrm{mM}$ Magnesium unless specified.

\section{Quantification of septin density on lipid monolayers}

The density of septins was estimated on shs1 complexes in interaction with lipid monolayer assays composed of $80 \%$ DOPC, $10 \%$ PS and $10 \%$ of PS, PI, PI(4)P, PI(4,5)P2 or PI(3,4,5,)P3. The experiments were carried without the presence of magnesium to prevent any assembly of shs1 complexes into high-ordered structures, and therefore promote the interaction of shs 1 complexes alone on the surface of the lipid monolayer. The resulting images were submitted to a trainable segmentation classification by using the plugin Weka [17] implemented in ImageJ. Since the staining is not identical from one image to another and can induce some bias in the contrast from one image to another, the software was trained to recognize the septins and the membrane for each image independently. The area covered by the septin complexes was assessed using ImageJ. 


\section{PI(4,5)P2 micelles experiments}

Five microliters of PI(4,5)P2 at $0.05 \mathrm{mg} \mathrm{ml}^{-1}$ was dried with argon and kept under vacuum for $1 \mathrm{~h}$. The dried lipidic film was re-suspended in $50 \mathrm{mM}$ Tris $\mathrm{pH} 8,150 \mathrm{mM} \mathrm{NaCl}$ to a final concentration of $0.1 \mathrm{mg} \mathrm{ml}^{-1}$. The lipid solution was gently sonicated for $30 \mathrm{~s}$ to get micelles. One microliter of Shs1 septin oligomers at $0.4 \mathrm{mg} \mathrm{ml}^{-1}$ was mixed with $2.5 \mu \mathrm{l}$ of micelles. The mixture was immediately diluted into $50 \mathrm{mM}$ Tris pH8, $2 \mathrm{mM} \mathrm{MgCl}_{2}$ to get a final protein concentration of $36 \mathrm{nM}\left(0.013 \mathrm{mg} \mathrm{ml}^{-1}\right)$ and a buffer $\sim 50 \mathrm{mM}$ Tris pH8, $20 \mathrm{mM} \mathrm{NaCl}, 1.8 \mathrm{mM} \mathrm{MgCl}_{2}$. The mixture was incubated for $1 \mathrm{~h}$ at $4^{\circ} \mathrm{C}$ before being negatively stained.

\section{Negative stain sample preparation and imaging}

Four microliters of diluted sample to a final concentration of $0.01 \mathrm{mg} \mathrm{ml}^{-1}$ were adsorbed for $30 \mathrm{~s}$ to $1 \mathrm{~min}$ on a glow-discharged carbon-coated grid (Electron microscopy Sciences, ref CF300-CU). The grids were then blotted, washed and finally negatively stained for $1 \mathrm{~min}$ with a drop of $2 \%$ uranyl formate at each step. The data were collected using either a Tecnai spirit microscope (Thermofischer, FEI, Eindhoven, the Netherlands) operated at an acceleration voltage of $80 \mathrm{kV}$ and equipped with a Quemesa (Olympus) camera or with a Lab6 G2 Tecnai (Thermofischer, FEI, Eindhoven, the Netherlands) operated at an acceleration voltage of $200 \mathrm{kV}$. The data were acquired with a $4 \mathrm{k} \times 4 \mathrm{k}$ F416 CMOS camera (TVIPS) in an automated manner using the EMTools software suite (TVIPS).

\section{Preparation of vesicles}

A lipid mix made of $70 \%$ DOPC, $10 \%$ DOPE, $10 \%$ DOPS and $10 \%$ of synthetic PI $(4,5) \mathrm{P} 2[18: 1]$ or PI $(3,4,5)$ P3 were first dried with argon and then kept under vacuum for an hour. The lipid film was re-suspended into $50 \mathrm{mM}$ Tris $\mathrm{pH} 8,300 \mathrm{mM} \mathrm{NaCl}$ to generate a heterogeneous mixture of vesicles with a final lipid concentration of $1 \mathrm{mg} \mathrm{ml} \mathrm{m}^{-1}$. Lipids and septin were incubated at a $10: 1$ mass ratio and diluted five times to obtain a final buffer with $50 \mathrm{mM}$ Tris pH8, $50 \mathrm{mM} \mathrm{NaCl}$. Vesicles and septins were incubated from $30 \mathrm{~min}$ to $1 \mathrm{~h}$.

\section{Cryo-tomography and tomogram processing}

Four microliters of samples supplemented with 10 -nm gold beads were adsorbed on lacey holey carbon grids (Ted Pella INC., Lacey grids). The grids were vitrified in liquid ethane using an automated plunge-freezing apparatus (EM-GP, Leica). The samples were analyzed at liquid nitrogen temperature using a Tecnai F20 (Thermofischer, FEI, Eindhoven, the Netherlands) microscope operated at $200 \mathrm{kV}$ and equipped with a Falcon II direct electron detector (Thermofischer, FEI, Eindhoven, the Netherlands). Tilt series were recorded in a bi-directional mode starting at angle 0 with an increment of $2^{\circ}$ to 60 and then to $-60^{\circ}$. The data were collected at an exposure of $1 \mathrm{e} / \AA$ at a nominal defocus varying from -2 to $-2.5 \mu \mathrm{m}$ and at a magnification of 50000 (except for the bundle's tomogram collected at a magnification of 25000 ). The acquisition was carried out at a pixel size of $4.26 \AA$ per pixels. Alignment of tilt series using gold fiducials and 3D reconstruction was performed by the etomo software [18].

\section{Tomogram segmentation and statistics}

Tomograms were segmented and analyzed using Amira 6.4 (Thermo Scientific Amira Software). Filaments were extracted using a dedicated template matching algorithm, accounting for the missing wedges effects induced by limited tilt angles [19], while membranes were segmented thanks to a membrane-enhancing filter [20]. The centerlines of both filaments, and membranes, were extracted to facilitate certain measurements and comparisons. Only filaments clearly interacting with the membranes, but not via their endings, were taken into account for the statistics calculations. Their curved length was computed as the length of their centerline representation, while the chord length corresponds to the length of the line segment joining their end-points. The average thickness of the filaments was measured from their voxel representation.

To characterize the interaction between the filament and the membranes, we relied on their respective centerline representations. The angle between filaments and membranes was computed, for each point of the filament, by finding the corresponding closest point on the nearest membrane and comparing both local orientations. Tomograms were segmented using the IMOD software. 


\section{Image processing}

Negatively stained images of Shs 1 protomers with a pixel size of $2.14 \AA$ were collected at a magnification of $50 \mathrm{~K}$ and $1.5 \mu \mathrm{m}$ underfocus with a Tecnai G2 20 Twin (FEI) and a TVIPS F416 camera CMOS $4 \mathrm{k} \times 4 \mathrm{k}$. Particle picking was performed using boxer from the EMAN software suite [21]. Particles of 203 pixels size were hand-picked. Particles were normalized against the background, classified and aligned to generate class averages using SPIDER [22]. One round of reference-free alignment and classification was performed before references were selected. Two rounds of multi-reference alignment and classification were performed thereafter. For WT complexes, 53 classes were generated from 2446 particles. For double linker mutants, 36 classes were generated from 2339 particles. For $\Delta 350$ mutants 24 classes were generated from 2829 particles. For $\Delta 350-445$ mutants 25 classes were generated from 2234 particles. For Lys36A mutants 34 classes were generated from 2374 particles. For Thr37V mutants 53 classes were generated from 2588 particles. From the different classes, the datasets were sorted by the number of subunits present within the complexes. The proportion of protomers within the dataset could thus be deduced from the number of particles per generated classes.

\section{Sequence alignment and homology structural analysis}

The alignment and homology investigation were carried out using software relying on a combination of structure and amino acid sequences analysis. Sequence alignment was operated using the structural alignment software Expresso [23] from the T-coffee server. Secondary structure predictions were performed using Quick2D from the MPI Bioinformatics Toolkit [24]. It has the advantage to display the results from different secondary structure prediction programs, allowing to control the accuracy of the predictions by redundancy. Notably, insights about secondary structures [25-30], disordered regions [31-33] and coiled-coils (CC) domains are generated [34-36]. CC domain predictions were further MULTICOIL2 [38] program. To further investigate the homology between proteins, especially to define the boundaries of the primary sequences used to obtain the subsequent models, a research and sequence alignment of homologous proteins, included distant ones, was conducted with HHPRED [37].

\section{Molecular modeling of GTP-binding domains of Cdc10, Cdc11 and Shs1 and docking of IP2}

To get a molecular three dimensional model of the GTPase domains of Cdc11, Shs1 and Cdc10, the I-tasser software [39] was used to predict the structure from the residue Lys18, just after the $\alpha 0$ helix, to Leu310 (Cdc11 numbering). A crystal structure was already solved for Cdc11 GTPase domain (PDB 5AR1), however, the flexible loops are missing [40]. Structures of septin GTP-binding domain, especially human septins, encompass these flexible parts, making modeling more accurate. Since Cdc11, Shs1 and Cdc10 auto-assemble via their NC-interface, dimers were built by fitting the model to septin 6 and 7 from the human septins 2-6-7 trimer solved by X-ray crystallography (PDB 2QAG). Finally, docking was performed using the SwissDock software [41], under default settings, between septin dimers and an IP2 molecule. Flexibility was allowed for the residues in the vicinity of the ligand with a maximum distance of $5 \AA$. A $20 \times 20 \times 20 \AA$ box was used for the docking, with Lys18 at its center. The docking experiment includes two basic residues essential for the interaction with PIP2 without considering the whole $\alpha 0$ helix (Lys18 and Arg19 for Cdc11). The different protein-ligand binding mode energies were calculated. The most favorable binding modes were clustered.

\section{Results}

Our report focuses on the behavior of Shs1 capped septin complexes, designated in the following as Shs $1_{\text {oct }}$. The experiments were led at least three times to ascertain their viability and reproducibility.

\section{Shs $1_{\text {oct }}$ in solution: a novel ultra-structural organization}

In vitro and in solution, the Shs1 octameric protomers, organized as Shs1-Cdc12-Cdc3-Cdc10-Cdc10-Cdc3Cdc12-Shs1, and designated Shs1 $1_{\text {oct }}$ are known to assemble into rods [7]. Above $150 \mathrm{mM} \mathrm{NaCl}$ we observed $36.1 \%$ octamers, $29.8 \%$ heptamers, 27.5\% hexamers and 6.6\% pentamers (Supplementary Figure S1). This polydispersity had already been documented previously [7] and accounts for a lower stability of the octameric Shs $1_{\text {oct }}$ complex as compared with the Cdc1 $1_{\text {oct }}$ complex. Within Shs $1_{\text {oct }}$, the Cdc12-Shs1 interface is thereby weaker than the $\mathrm{Cdc} 12-\mathrm{Cdc} 11$ interface in the $\mathrm{Cdc} 11_{\text {oct }}$. Indeed, the expression and purification of recombinant $\mathrm{Cdc} 11_{\text {oct }}$ generate almost $100 \%$ of intact octameric complexes [14]. At lower ionic strength $(20 \mathrm{mM} \mathrm{NaCl}$, $2 \mathrm{mM} \mathrm{MgCl} 2$ ) micrometric rings made out of bundled filaments were assembled (Figure $2 \mathrm{~A}$ ) as previously 


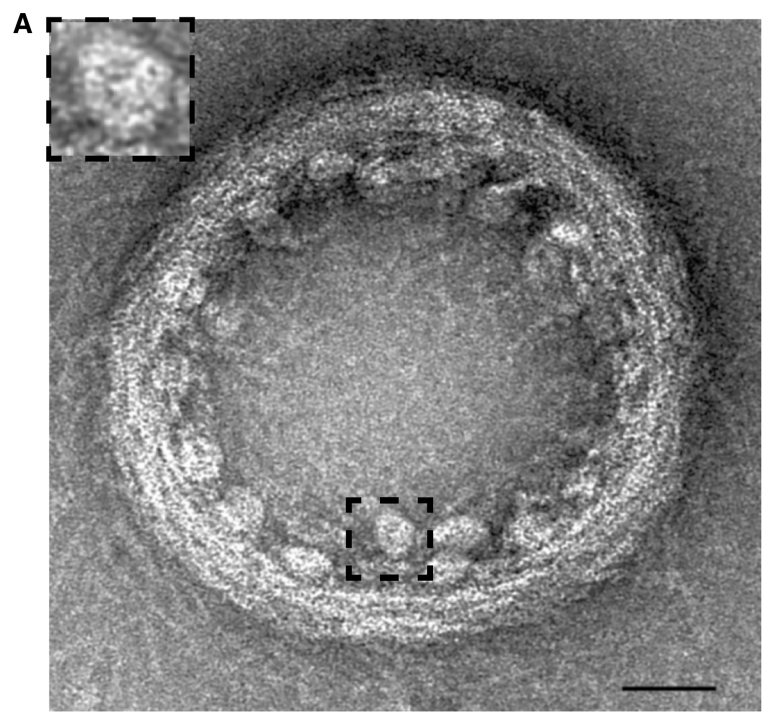

B
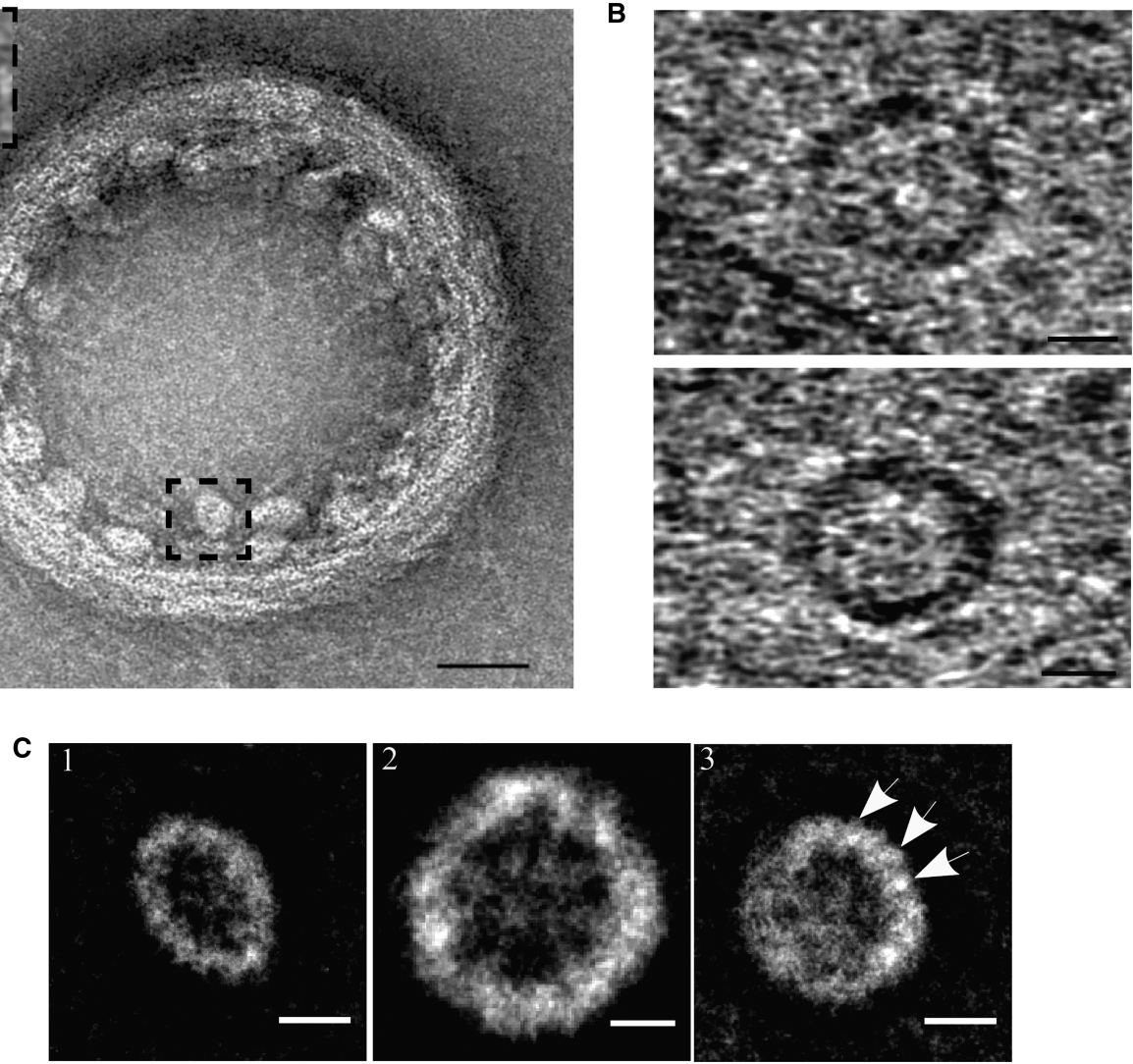

Figure 2. Self-assembly of Shs1 into nanometer wheels-like structures in solution.

(A) Ring-like structures of Shs $1_{\text {oct }}$ in low salt conditions. Small rounded-shaped densities are displayed against the inner surface of the ring (square dotted insert). Scale bar: $100 \mathrm{~nm}$. (B) Wheels observed in frozen-hydrated conditions at the vicinity of a bundle of Shs1 $1_{\text {oct }}$. Those wheels were isolated from the cryo-tomogram in Supplementary Movie S1. Scale bar: $10 \mathrm{~nm}$. (C) Three class averages of the wheels. Both ovoid- and rounded-shape wheels were observed (respectively 1 and 2). Small 'beads' (arrows) and blurry connecting densities in the middle of the wheels resemble domain organization of septin subunit namely GDB and CTE domains, respectively. Scale bars: $10 \mathrm{~nm}$.

reported. It was previously described that those micrometric bundled ultra-structures were assembled exclusively from intact octameric Shs1 capped oligomers [7] while the truncated oligomers were left out in solution.

Surprisingly, smaller structures were visible as well at low salt concentrations, but are absent from high salt concentration where the Shs1-Shs1 end-to-end association is impeded. These assemblies are circular structures of $\sim 100 \mathrm{~nm}$ in diameter. They were usually seen in the vicinity of straight or curved bundles (Figure 2A, boxed dotted square). Those peculiar ultra-structures were present in both negatively stained samples (Figure 2A) and frozen-hydrated preparations (see corresponding enlargements in a slice of a cryo-tomogram displayed in Figure 2B and Supplementary Movie S1). These are, therefore, not artifacts from sample preparation resulting from drying or staining of the samples. Even though those peculiar assemblies are clearly visible in the figures of published reports focusing on human septins as well and thus suggesting those structures are conserved $[7,42]$, they had not been commented. Two-dimensional averaging and classification were performed. The 'wheel-like' structures are heterogeneous both in size and shape, suggesting that their assembly is adjustable. Indeed, some 'wheels' were ovoidal (Figure 2C.1) while others were more circular (Figure 2C.2). In Figure 2C.3 (white arrows), densities corresponding to septin subunits are unambiguously displayed. Each of the densities were measured to be $5.4 \pm 1.1 \mathrm{~nm}(n=67)$, corresponding to the size of the septin globular domain. The diameter of the wheels were measured at $21.2 \pm 2.7 \mathrm{~nm}(n=112)$, leading to a perimeter of $\sim 60 \mathrm{~nm}$, the equivalent of two rods interacting via their Shs1 subunits. Within the inner portion of the 'wheels', some protein filamentous densities connecting opposite subunits are visible. We suspect that these densities are composed of flexible 
CTEs from Shs1, Cdc12 and Cdc3 which would be involved in the stability of those 'wheels'. Indeed, the globular domains are unambiguously present on the periphery of this peculiar structure and the domains contributing to the density pointing inwards towards the center of the structure can only result from the contribution of the flexible CC domains.

Septins are known to interact with membranes doped with phosphoinositides [12,43,44]. In particular, budding yeast septins interact with $\mathrm{PI}(4,5) \mathrm{P}_{2}[12,14]$. Cdc1 $11_{\text {oct }}$ polymerization was shown to be facilitated on lipid monolayers doped with $\mathrm{PI}(4,5) \mathrm{P}_{2}$ exclusively [14] and to induce liposomes deformations [13]. Cdc1 $11_{\text {oct }}$ indeed assemble into micron-scale filaments even in non-polymerizing conditions. For the first time, we determined how Shs1 protomers assemble on the surface of biomimetic membranes doped with $\mathrm{PI}(4,5) \mathrm{P}_{2}$.

\section{Shs1 protomers display a high polymorphism when interacting with lipid monolayer doped with PI(4,5)P2}

Most of the experimental conditions used throughout the lipid monolayer assays are identical $(36 \mathrm{nM}(0.01 \mathrm{mg}$ $\mathrm{ml}^{-1}$ )) final protein concentration, $4^{\circ} \mathrm{C}$ and overnight incubations and a minimal lipid composition of $80 \%$ DOPC, $10 \%$ DOPS and 10\% phosphoinositides). An addition of nucleotides, salt and the nature of the phosphoinositides varied, when indicated.

On lipid monolayers doped with $\mathrm{PI}(4,5) \mathrm{P}_{2}$, shs1 complexes assemble into a wide variety of high-ordered structures. To enhance the clarity of the corresponding figure, Figure 3, the images have been duplicated to segment septin densities. Shs $1_{\text {oct }}$ assemble into interconnected rods $(100 \mathrm{~nm}$ long at most), within a 2D network (Figure 3A). This homogeneous network of interconnected septin rods covers the surface. In addition, peculiar bundled assemblies of filaments are observed bound to this organized 2D network carpet of Shs $1_{\text {oct }}$ protein. Out of 149 bundled dense structures reported, 82\% $(n=122)$ were micrometric bundled rings (Figure 3B). The rings were similar to those obtained in solution, with comparable dimensions (mean outer diameter $326 \pm 62 \mathrm{~nm}$, mean inner diameter $200.5 \pm 42 \mathrm{~nm}(n=49))$. Some rings revealed, on their inner
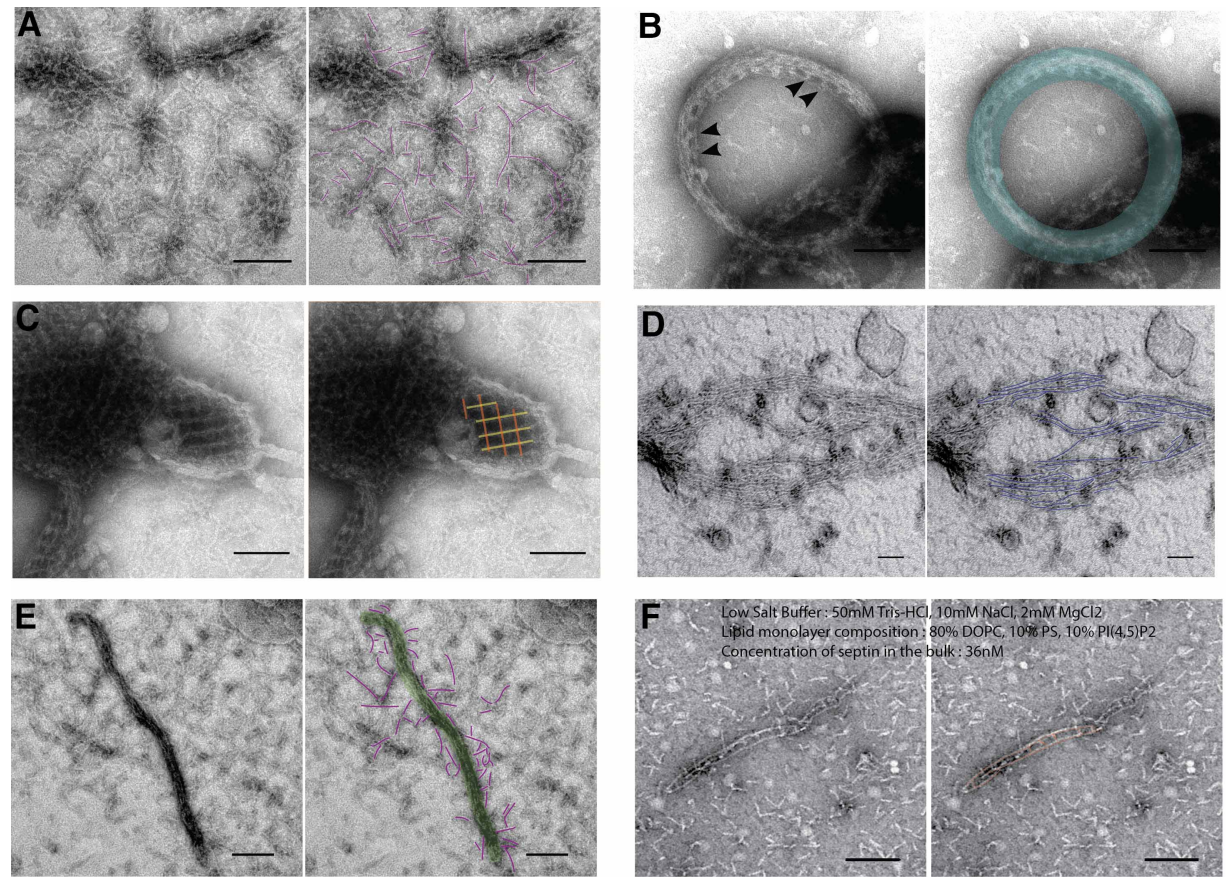

G

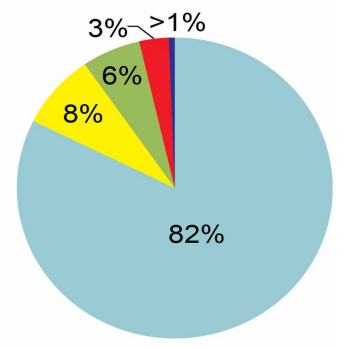

Rings

Gauzes

Bundles

Paired filaments

Sheets of spaced filaments

Figure 3. Shs $1_{\text {oct }}$ self-assembly bound to a PI(4,5)P2 doped lipid monolayer.

Architectures of Shs $1_{\text {oct }}$ oligomerized on lipid monolayer doped with $10 \%$ of $\mathrm{PI}(4,5) \mathrm{P}_{2}$ : $2 \mathrm{D}$ network of short filaments $(\mathbf{A})$, rings $(\mathbf{B})$, gauzes $(\mathbf{C})$ and sheet of spaced and long filaments (D), thin bundles of tightly packed filaments $(\mathbf{E})$ and paired filaments (F). The ratio of each type of architecture is displayed on the chart (G). Gauzes are characterized by larger (yellow) and thinner (orange) densities. The compositions of the lipid monolayer ((80\% DOPC, 10\% DOPS, $10 \% \mathrm{PI}(4,5) \mathrm{P}_{2}$, buffer $\left(50 \mathrm{mM}\right.$ Tris- $\left.\mathrm{HCl} \mathrm{pH} 8,10 \mathrm{mM} \mathrm{NaCl}, 2 \mathrm{mM} \mathrm{Mg}{ }^{2+}\right)$ as well as the concentration of shs $1_{\text {oct }}(36 \mathrm{nM})$ are displayed directly on the figure. Scale bars $=100 \mathrm{~nm}$. 
surface, a proteic density displaying a periodicity of $23.6 \pm 3.9 \mathrm{~nm}(n=62, \min =13.5 \mathrm{~nm}, \max =31.4 \mathrm{~nm})$ and most likely resulting from extending flexible rather disorganized coiled coils. (Figure 3B, arrows).

In addition to ring-like structures, to a lesser extent, gauzes of orthogonal filaments represented $8 \%$ of the ultra-structures (Figure 3C). Surprisingly, on $\mathrm{PI}(4,5) \mathrm{P}_{2}$ doped monolayers, the formation of gauzes is independent on the phosphorylation state of Shs1, in contrast with previous observations in solution [7]. Importantly, $S h s 1_{\text {oct }}$ complexes were expressed in E. coli which minimizes post-translational modifications [45]. In Garcia et al. gauzes were observed exclusively with a specific phosphomimetic mutant of Shs1, not with wild type proteins. The gauzes obtained in our conditions are always connected and juxtaposed to another ultrastructure, for instance, embedded within a ring-like ultrastructure (Figure 3C).

Other higher-ordered structures were observed: sheets of loose and parallel spaced filaments (Figure 3D), bundles of tight filaments (6\%, Figure 3E) and isolated paired filaments (3\%) (Figure 3F). The ultrastructure of Shs $1_{\text {oct }}$ is thus polymorphic (Figure 3G). The higher-order structures are most likely composed of octameric intact complexes while the $2 \mathrm{D}$ carpet of proteins would gather the hexameric and heptameric truncated septin rods. Indeed, those ultra-stuctures (rings, bundles) are assembled from the intact octameric Shs1 capped oligomers while the shorter oligomers are left out as individual connected protomers, as already analyzed by singleparticle analysis [7]. Previously, we had demonstrated that hexameric Cdc11 less septin rods can assemble into a connected network of filaments, bound to a PI(4,5)P2 doped lipid monolayer [14], while no other higherordered structures were generated. Hence, the similar observed 2D network here (Figure 3A) most likely draws most of the destabilized shorter septin complexes.

\section{Shs $1_{\text {oct }}$ septin complexes interact with lipid monolayers doped with different charged lipids}

Septins are known to interact with membranes doped with phosphoinositides [12,43,44]. In particular, budding yeast septins interact with $\mathrm{PI}(4,5) \mathrm{P}_{2}$ in a specific manner $[12,14]$ and interacts poorly with other charged lipids (DOPS) and phosphoinositides. The interaction of $\mathrm{Cdc1} 1_{\text {oct }}$ with $\mathrm{PI}(4,5) \mathrm{P}_{2}$ was already thoroughly characterized and shown to be primarily mediated by Cdc10. Because both $\mathrm{Cdc1} 1_{\text {oct }}$ and Shs $1_{\text {oct }}$ are present within the hourglass-like assembly of septins at the collar bud neck of $S$. cerevisiae during mitosis [7], we addressed whether $S h s 1_{\text {oct }}$ interacted with $\mathrm{PI}(4,5) \mathrm{P}_{2}$ as well.

After incubating unpolymerized Shs $1_{\text {oct }}$ with pure micelles of $\mathrm{PI}(4,5) \mathrm{P}_{2}$, the resulting bundles were decorated in a periodic fashion (Figure $4 \mathrm{~A})$. A repetitive spacing of $16.4 \pm 1.2 \mathrm{~nm}$, approximately half an octamer, $(n=10$, arrows, Figure 4A), with a center to center distance of $29.4 \pm 0.8 \mathrm{~nm}$ was observed. Besides, incubated with LUVs (large unilamellar vesicles) doped with $\mathrm{PI}(4,5) \mathrm{P}_{2}$, the Shs $1_{\text {oct }}$ were occasionally bound to liposomes by their ends (hence through the Shs1 subunit) as filamentous short brushes (Figure 4B, middle and Supplementary Movie S2). In addition, tethered vesicles, connected by a high density of septin rods, thus organized in 'brushes' (Figure 4B, bottom) were observed. Septins are curvature sensitive and they sense micrometric curvatures $[13,46]$. Septin filaments tend to avoid interacting with highly curves structures (i.e. small vesicles of less than $100 \mathrm{~nm}$ in diameter) while they bind to vesicles displaying diameters of $500 \mathrm{~nm}$ or more and flat surfaces. Shs1 capped septins can bind to vesicles using two different strategies. Either they bind by their ends (shs1 subunit) or they lie flat on lipids interacting along their whole length (through a Cdc10 mediated interaction). In particular, septins cannot interact laterally (along the length of filaments) with small vesicles which are by definition highly positively curved. Thereby, interacting via their ends is most likely more favorable energetically on small vesicles. The diameters of vesicles decorated by a 'brush-like pattern' of Shs $1_{\text {oct }}$ were indeed measured to be $26.9 \pm 20.5$ as compared with $322 \pm 229 \mathrm{~nm}$ for naked vesicles (see Figure $4 \mathrm{~B}$ ). Taken together, both of the aforementioned observations suggest that $\mathrm{PI}(4,5) \mathrm{P}_{2}$ micelles bind preferentially both Shs1 (end subunit) and Cdc10 (subunit in the center) in the complex.

We addressed the affinity specificity of $\mathrm{Shs} 1_{\text {oct }}$ for $\mathrm{PI}(4,5) \mathrm{P}_{2}$ as compared with other charged lipids (Figure 4C). On monolayers doped with $20 \%$ PS or $10 \%$ of PI a few isolated octamers were visualized. However, Shs1 $1_{\text {oct }}$ did not interact at all with an uncharged monolayer (DOPC only). The network-like organization of Shs1 $1_{\text {oct }}$ rods on lipid monolayers was visualized in the presence of phosphoinositides or in the presence of PS (Figure 4C, lower panel: $10 \% \mathrm{PI}(4,5) \mathrm{P}_{2}$ ). The density of septin complexes has been quantified on electron microscopy images to compare the interaction of Shs $1_{\text {oct }}$ with various lipids. Using algorithms based on deep learning, the filamentous protein within each image has been segmented to determine de density of complexes (see Method section and Supplementary Figure S2). The results are displayed in Figure 4C. Doping 

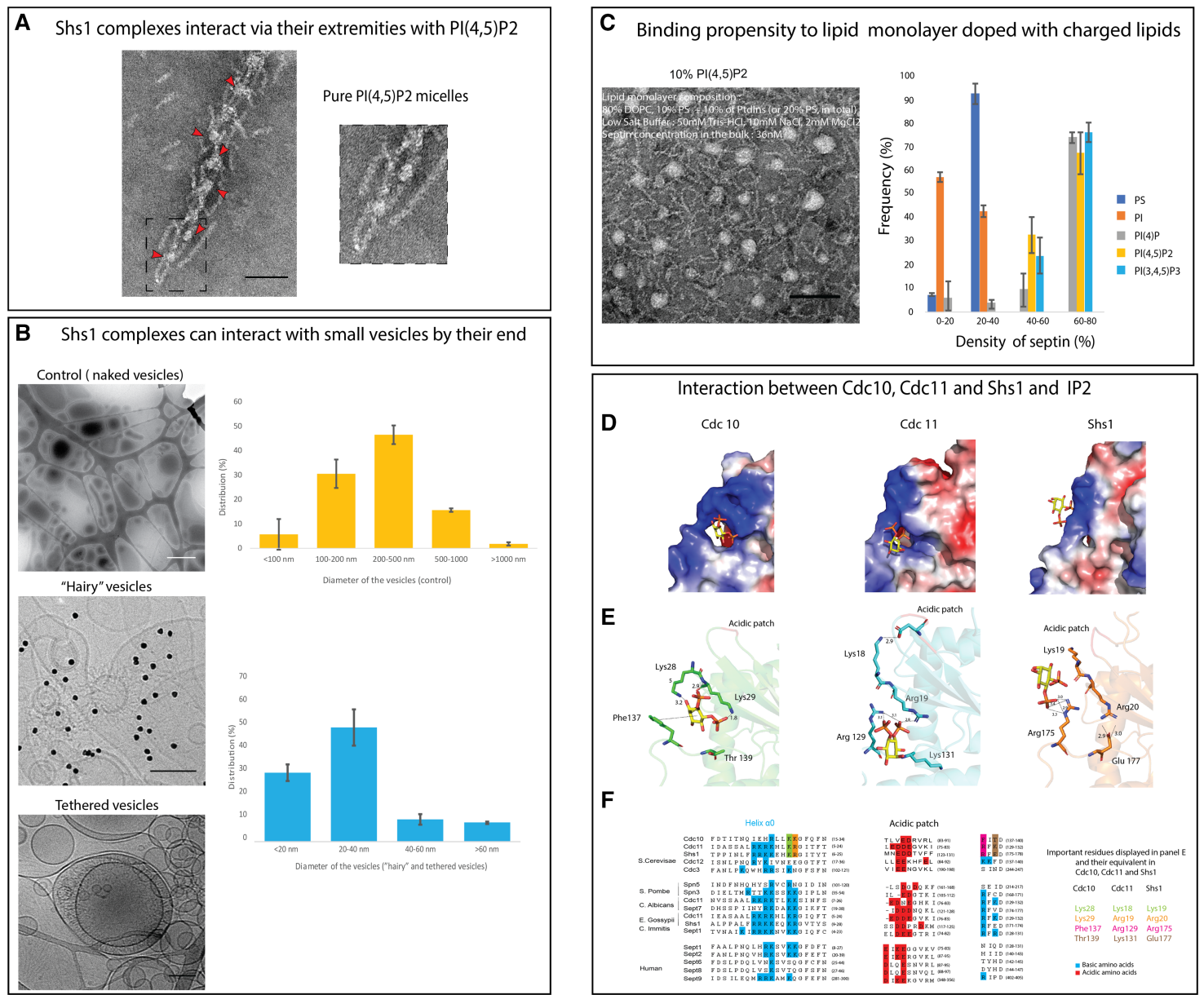

Figure 4. Shs $1_{\text {oct }}$ interaction and binding with lipids.

(A) Shs1 oct bundle interacting with $\mathrm{PI}(4,5) \mathrm{P} 2$ micelles (red arrows): periodic decoration. Scale bar $=20 \mathrm{~nm}$. (B) left panel: images from cryo-AM samples. Top: control vesicles. Scale bar: $1 \mu \mathrm{M}$, middle: Brushes of septin rods bound to vesicles. Scale bar: $50 \mathrm{~nm}$, bottom: tethered vesicles by septin rods. Scale bar: $100 \mathrm{~nm}$. The black dots are $10 \mathrm{~nm}$ in diameter gold beads used as fiducials to perform cryo-electron tomography. Right panel: diameter distribution of the control vesicles (orange, top) and of tethered and brush-covered vesicles (blue, bottom). (C) Left panel: Shs $1_{\text {oct }}$ complexes incubated with lipid monolayers doped with, $10 \% \mathrm{PI}(4,5) \mathrm{P}_{2}$. It has to be noticed that no magnesium was used to avoid any polymerization of shs $1_{\text {oct }}$ and thus evaluate properly its propensity of binding to different lipid monolayers. An example of such organization is display on negative staining image on the left of the panel $(10 \% \mathrm{PI}(4,5) \mathrm{P} 2)$. Scale bars: $100 \mathrm{~nm}$. Right panel: the density of septins on the surface of each monolayer doped with DOPS (blue), PI (orange), PI4P (gray), PI(4,5)P2 (yellow) or PI(3,4,5)P3 (cyan) was measured by deep learning segmentation, and were reported in the graphs. (D,E) Molecular docking of IP2 molecules (yellow) within models of GBD septin domain of Cdc10 (left), Cdc11 (middle) and Shs1 (right) subunits. (D) Electrostatic representation. (E) Atomic representation. The most stable IP2 location was displayed for each septin. For each subunit, the relevant acidic patch able to potentially recruit basic residues interacting with IP2 is colored in red. The distances between the residues (in Angstrom) are specified in (E). The numbering refers to each septin specifically. (F) Sequence alignments comparing S. cerevisiae mitotic septins, S. Pombe, C. albicans, E. Gossypii, C. Immitis and Human septins. The blue residues are basic residues. Red residues are acidic. The important residues displayed in the atomic representation of the IP2 binding pocket in (E) were listed on the right of the sequence alignment to facilitate the comparison of the docking results. Lys $28^{\text {cdc10 }}$ and its corresponding

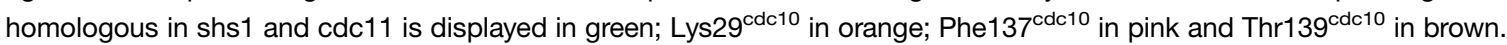
Those residues were also highlighted in the sequence alignment, using the same color code aforementionned. 
the lipid monolayer with $\mathrm{PI}(4) \mathrm{P}, \mathrm{PI}(4,5) \mathrm{P} 2$ or $\mathrm{PI}(3,4,5) \mathrm{P}$ results in enhancing the density of septin filaments as compared with PI or PS.

Taken together, our observations suggest that the Shs $1_{\text {oct }}$ interacts with phosphoinositides primarily via electrostatic interactions and is much less specific to $\mathrm{PI}(4,5) \mathrm{P}_{2}$ than the interaction of $\mathrm{Cdcl}_{\text {oct }}$ with $\mathrm{PI}(4,5) \mathrm{P}_{2}[12,14]$.

The interaction of $c d c 11_{\text {oct }}$ with membranes is primarily driven by the cdc10 subunit [14]. However, in shs1 $1_{\text {oct }}$, both shs1 and cdc10 subunit are able to interact with $\mathrm{PI}(4,5) \mathrm{P} 2$. To investigate the rationale for such differences, we have carried out a local and flexible docking (for the residues in the vicinity of the IP2 molecule up to $5 \AA$ ) between an IP2 molecule and the binding pockets of Cdc10, Cdc11 and Shs1 GBDs models (Figure 4) using I-TASSER [39]. The resultant fullfitness and $\Delta G$ values are presented in Table 1. In addition, a representation of the electrostatic surfaces in the vicinity of the IP2 binding pocket has been added.

The binding pocket to IP2 is predominantly charged positively for both cdc10 and cdc11 while the binding pocket of shs1 is characterized by a smaller positively charged surface (Figure 4D). Moreover, the space available for the IP2 molecule is larger in cdc10 than cdc11 and shs1. Thus, the IP2 molecule fits within the binding pocket of $\operatorname{cdc} 10$, whereas the ligand is not entirely embedded for $\operatorname{cdc} 11$, and clearly pushed away for shs1. In the case of shs1, the IP2 molecule is thus constrained to interact with the charged surface, which dimension is reduced. The IP2 is thereby displaced as compared with the configuration observed with Cdc10 and Cdc11, explaining why it is characterized by the lowest affinity (Table 1).

In agreement with previous studies, Cdc10 had the highest theoretical affinity for IP2 molecules. The two lysines able to sustain the interaction between phospholipids and septins in vivo, Lys $28_{\mathrm{cdc1} 10}$ and Lys $29_{\mathrm{cdc1} 10}$, are involved in the correct positioning of the IP2 molecule (Figure 4E, left). Interestingly, the residue Phe $137_{\text {cdc10 }}$ is engaged in a cation-pi interaction with Lys $28_{\mathrm{cdc10}}$ and therefore stabilizes its orientation within the IP2 binding pocket.

Even though both of these lysines are chemically conserved, Cdc11 and Shs1 displayed the lowest theoretical affinity for IP2 molecules (see Table 1). Strikingly, the equivalent residues for Lys $28_{\text {cdc10 }}$ (Lys $18_{\text {cdc11 }}$ and Lys $19_{\text {shs } 1}$ ) do not interact with IP2 in the generated model. Both shs1 and cdc11 subunits exhibit an arginine substituting the phenylalanine Phe137 ${ }_{\mathrm{cdc} 10}\left(\mathrm{R} 129_{\mathrm{cdc1} 1}\right.$ and $\mathrm{R} 175_{\mathrm{shs} 1}$ on Figure $\left.4 \mathrm{E}\right)$. Also, both Lys $18_{\mathrm{cdc} 11}$ and Lys $19_{\text {shs1, }}$, corresponding to Lys $28_{\text {cdc10 }}$, are pointing toward an acidic patch (colored in red in Figure 4E). In addition, Lys18 $8_{\mathrm{cdc11}}$ interacts with the carboxyl functional group of an aspartate, whereas Lys $19_{\text {shs } 1}$ interacts with the backbone of the loop. Therefore, it is more likely that R129 cdc11 $_{11}$ and R175 $5_{\text {shs } 1}$, replacing Phe137 ${ }_{\text {cdc10 }}$, disturb the orientation of the Lys $18_{\mathrm{cdc11}}$ and Lys $19_{\text {shs1 }}$ which is thus constrained and forced to interact with the acidic patch. R129 $9_{\mathrm{cdc} 11}$ and $\mathrm{R} 175_{\mathrm{shs} 1}$, by replacing Phe137 ${ }_{\mathrm{cdc} 10}$ and precluding the interaction between Lys $18_{\text {cdc1 } 1} /$ Lys $19_{\text {shs } 1}$ and the substrate, induce a dramatic changing in the position of the IP2 molecule. The loop containing the acidic patch is not conserved in sequence and in length, but displays aspartates and glutamates residues (see sequence alignment in Figure 4). Given this part of the protein was considered as part of the rigid block, Lys $28_{\mathrm{cdc} 10}$ and Lys $19_{\text {shs } 1}$ potentially interacts specifically with the functional groups of this patch.

Shs1 displayed the lowest theoretical affinity for IP2 molecules (see Table 1). Out of the three basic residues present in the binding pockets of Cdc11 and Shs1, only Arg175 shs1 is involved in an interaction with IP2 (equivalent to $\operatorname{Arg} 129_{\mathrm{cdc1}}$ and Phe137 ${ }_{\mathrm{cdc1}}$ ), against two basic residues for cdc11. Because $\operatorname{Arg} 20_{\text {shs }}$ is involved

Table 1. Fullfitness and Estimated $\Delta G$ values of the three best matches concerning the cluster 0 obtained for cdc10, cdc11 and shs1 GBD docking with an IP2 ligand

\begin{tabular}{lllll}
\hline $\begin{array}{l}\text { Septin } \\
\text { subunit }\end{array}$ & $\begin{array}{l}\text { No. of SwissDock } \\
\text { Clusters }\end{array}$ & $\begin{array}{l}\text { Cluster rank from } \\
\text { cluster } \mathbf{0}\end{array}$ & $\begin{array}{l}\text { Fulfitness (kcal/ } \\
\text { mol) }\end{array}$ & $\begin{array}{l}\text { Estimated } \Delta \mathbf{G} \text { (kcal/ } \\
\text { mol) }\end{array}$ \\
\hline \multirow{2}{*}{ cdc10 } & 48 & 0 & -2494.9043 & -15.615602 \\
& & 1 & -2494.9043 & -15.615602 \\
& & 2 & -2494.9043 & -15.615602 \\
cdc11 & 48 & 0 & -2736.9548 & -15.8689 \\
& & 1 & -2696.602 & -10.595408 \\
shs1 & 2 & -2684.1865 & -8.829166 \\
& 48 & 0 & -2883.427 & -7.783838 \\
& & 1 & -2882.1416 & -7.7561283 \\
\hline
\end{tabular}


in an interaction with a glutamate, Glu177 ${ }_{\text {shs } 1}$, its interaction with the IP2 molecule is precluded. Cdc10 and

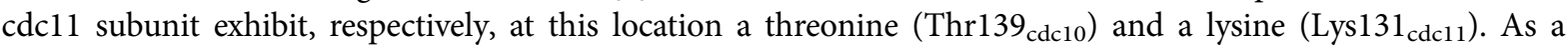
result, Arg20 $0_{\text {shs } 1}$ does not interact with the ligand as its equivalent Arg19 ${ }_{\text {cdc11 }}$ and Lys $28_{\text {shs } 1}$.

Therefore, in Cdc11 and Shs1, fewer residues could be involved in the IP2 interaction, suggesting that the cdc10 subunit could have a higher affinity for PIP2 molecule in vitro [14]. The simulated model could explain why Shs1 capped complexes interact less specifically with IP2 than Cdc11 capped complexes.

\section{Role of the C-terminal extension (CTE) on Shs1 oct $_{\text {assembly }}$}

$\mathrm{Cdc} 11_{\text {oct }}$ and Shs $1_{\text {oct }}$ organize differently bound to a lipid monolayer doped with $\mathrm{PI}(4,5) \mathrm{P}_{2}$. When comparing the Cdc11 and Shs1 subunit sequences, their CTE differs the most significantly. To understand whether the CTE of Shs1 plays a role in the organization of $S h s 1_{\text {oct }}$ on lipid monolayers, different mutants affecting the CTE of Shs1 were designed.

The CTE domain encompasses a flexible linker and a predicted CC domain (Figure 5A). We have designed several mutants with either deletions or insertions within the CTE of the Shs1 subunit to test for its function into Shs $1_{\text {oct }}$ assembly. We truncated both the CC domain and the linker upstream the CC (Shs1 ${ }^{\Delta 350}$ ), the linker only (Shs $\left.1^{\Delta 350-445}\right)$ and a third mutant with twice the linker length (Shs $1^{\times 2 \text { linker }}$ ).

By 2D image analysis, we determined the stability of the generated mutants in high salt conditions (Figure 5B). As compared with the wild type protein, the truncated mutants were significantly more
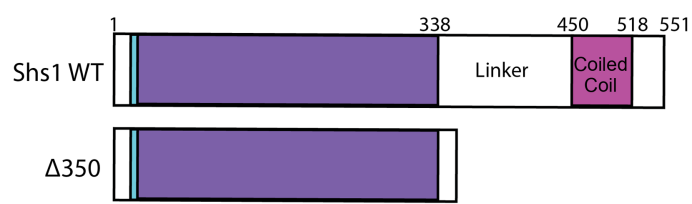

$\Delta 350-445$

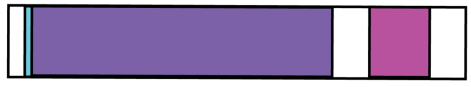

x2 linker

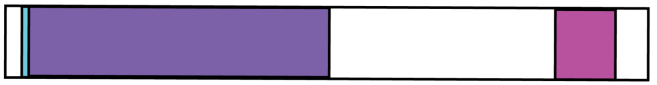

B

Size distribution of shs 1 WT and CTE mutants oligomers in solutio High Salt Condition (30mM Tris pH 8, 300mM NaCl, 2mM MgCl2)

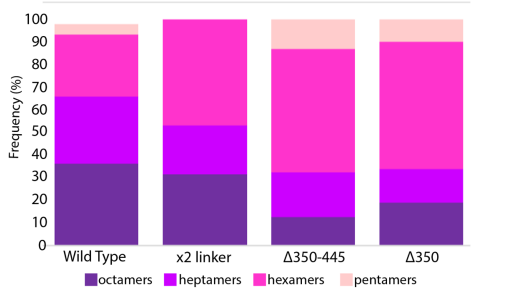

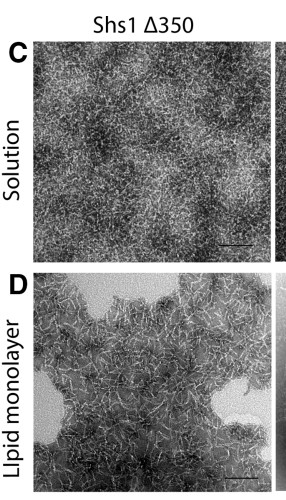

Shs1 $\Delta 350-445$
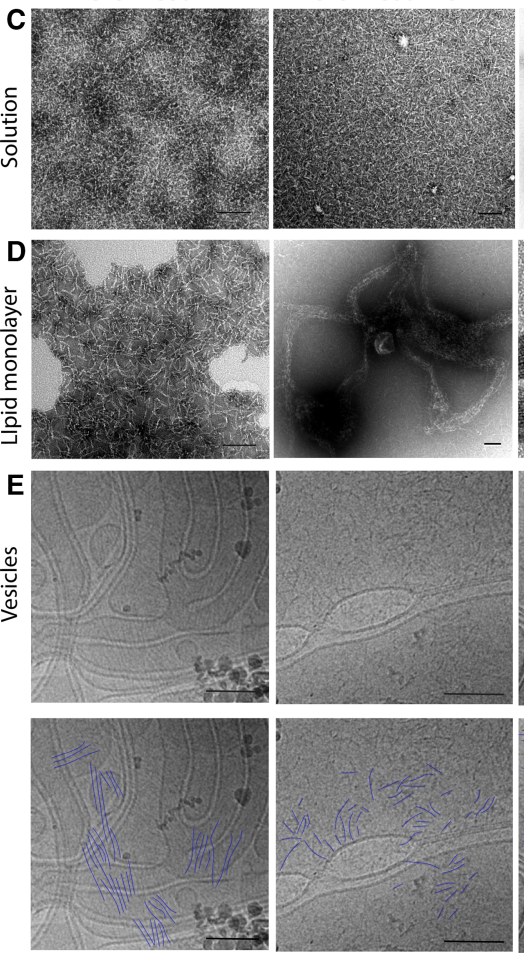

Shs $1 \times 2$ linker
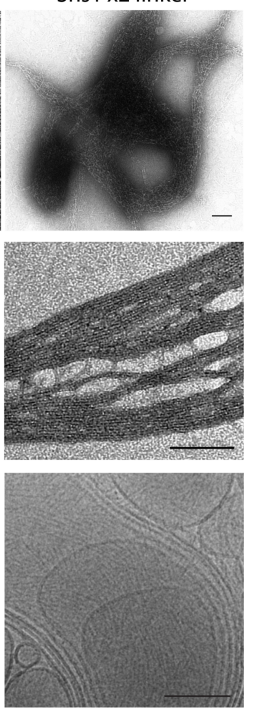
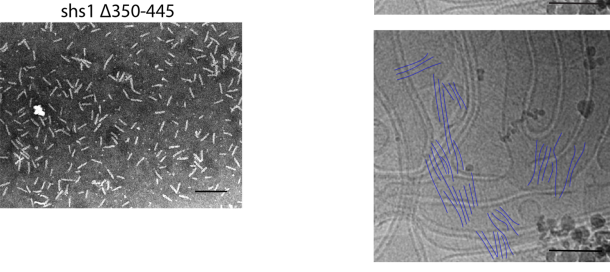

shs $1 \Delta 350$

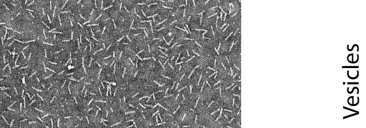

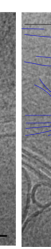

Figure 5. Ultrastructure of CTE Shs1 mutants within septin complexes Shs1 $1_{\text {oct- }}$

(A) Schematic representation of the different constructs used to assess the role of the CTE domain. (B) Size distribution of Shs1 ${ }_{\text {oct }}$ oligomers (Shs $1_{\text {oct }}$, Shs $1^{\Delta 350-445}$ oct, Shs $1^{\Delta 350}$ oct and Shs $1^{\times 2 \text { linker }}$ oct). Representative electron microscopy image of Shs $1^{4350}$ oct (top) and Shs $1^{\Delta 350-445}$ oct (bottom) used for 2D image processing. Scale bars: $100 \mathrm{~nm}$. (C) Self-assembly of Shs $1^{\Delta 350}$ oct (left, scale bar: $200 \mathrm{~nm}$ ), Shs $1^{\Delta 350-445}$ oct (middle, scale bar: $500 \mathrm{~nm}$ ) and Shs $1^{\times 2 l i n k e r}$ oct (right, scale bar: $100 \mathrm{~nm}$ ) in solution and low salt conditions. (D) On a lipid monolayer doped with 10\% PI(4,5)P2, Shs $1^{\Delta 350}$ oct assembles into a network of rods (left), Shs $1^{\Delta 350-445}$ oct into bundled structures (middle) and Shs $1^{\times 2 \text { linker }}$ oct into sheets of parallel and tightly packed filaments (right). Scale bars: $100 \mathrm{~nm}$. (E) On liposomes doped with $10 \%$ of PI(4,5)P2, Shs $1^{\Delta 350}{ }_{\text {oct }}$ assembles into spaced and parallel filaments (left), Shs $1^{\Delta 350-445}$ oct into rather short and disorganized filaments (middle) and Shs $1^{\times 2 \text { linker }}$ oct into long and parallel filaments (right). Scale bars: $100 \mathrm{~nm}$. On the lower panel, septin filaments are highlighted in blue to enhance visibility. 
destabilized, with the proportion of intact octameric complex reduced to $\sim 20 \%$. However, Shs $1^{\times 2 \text { linker }}$ was as stable as the wild type complex. Hence the flexible CC domain has a role in the stabilization of the complex. In solution, and polymerizing conditions (low ionic strength), the Shs $1_{\text {oct }}^{\Delta 350}$ and Shs $1^{\Delta 350-445}$ oct did not assemble into long filaments or higher-ordered structures (Figure 5C). No wheel-like small complexes (see Figure 2) were generated. Only rod-like assemblies were visualized showing the essential role of the coiled coils of Shs1 for polymerization and bundling (Figure 5C, left panel $\left(\mathrm{Shs}_{1}^{\Delta 350}{ }_{\text {oct }}\right)$ and middle panel $\left(\mathrm{Shs}^{\Delta 350-445}{ }_{\text {oct }}\right)$ ). Shs1 with twice the linker (Shs1 ${ }^{\times 2 \text { linker }}{ }_{\text {oct }}$ ) was able to form bundled rings similar to those obtained with the wild type septin complex (Figure 5C, right panel).

On lipid monolayers, the truncation mutants mostly interacted with monolayers as a network of interconnected rods (Figure 5D, left panel) and occasionally self-assembled into bundled structures (Figure 5D, middle panel) which were, however, rather disordered. Bound to vesicles, the truncated mutants also self-assemble into polymerized rods (Figure 5E), suggesting the mutations do not prevent polymerization to occur. To some extent, the interaction of truncated mutants with lipids thus enhanced the self-assembly of septin complexes into bundled ultra-structures (Figure 5D). Shs1 with twice the linker $\left(\mathrm{Shs} 1^{\times 2 \text { linker }}\right.$ ) was able to self-assembled into micron-length organized sheets of tight parallel filaments (Figure 5D, right panel), mimicking Cdc11 assembly on the surface of a lipid monolayer [14].

\section{Cryo-electron tomography reveals a highly similar organization of Shs1 ${ }_{\text {oct }}$ and Cdc11 oct $_{\text {on }}$ deformed liposomes}

Vesicles incubated with $S h s 1_{\text {oct }}$ were strongly aggregated by the interacting protein complex. Shs1 capped septins can bind to vesicles using two different strategies. Either they bind by their ends (shs1 subunit, Figure 4B) or they lie flat on lipids interacting along their whole length (Figure 6 and Supplementary Movies S2 and S3). The vesicles covered with straight septin filaments were flattened in addition to displaying local distortions: protrusions (Figure 6B, bottom left) and sharp or smooth tips (Figure 6B, upper right and bottom left). Measured on the 3D reconstructions, the thickness of the flattened vesicle varies between 120 and $45 \mathrm{~nm}(n=15)$, (including the protrusions which displays a smaller thickness between 90 and $45 \mathrm{~nm})$ as compared with $150-200 \mathrm{~nm}(n=7)$ obtained usually for naked control vesicles. The flattening could result from both the deformability of the
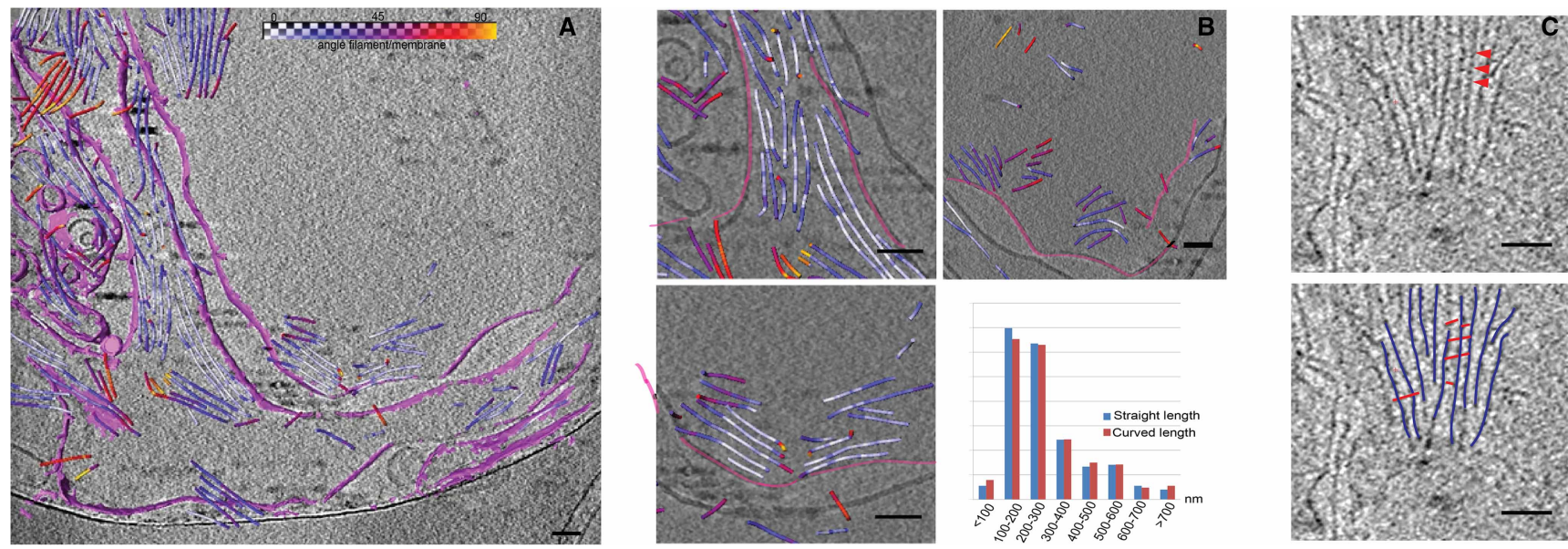

Figure 6. Organization, orientation of Shs $1_{\text {oct }}$ bound to deformed vesicles.

(A) Shs1 complexes in interaction with vesicles. Slice of tomogram extracted from the tomogram displayed in Supplementary Movie S2. The vesicles are segmented in pink, whereas septin proteins are colored following the color code at the top of panel (A). (B) Shs1 complexes distort membranes (tomogram slices extracted from the tomogram display in movie1). Septin filaments are segmented and color coded with their relative orientation to the edges of the vesicle membranes (see color code top of panel (A)). The angle between the nearest edge of the membrane and septin filament was calculated and thus reported. The length distribution of the filaments is reported in the chart (bottom right). Scale bars $=$ $100 \mathrm{~nm}$. (C) Shs $1^{\mathrm{WT}}$ assemble into sheet of spaced parallel filaments connected laterally by repetitive orthogonal densities (red arrowheads, left bottom panel) Scale bars $=100 \mathrm{~nm}$. 
membrane and the intrinsic properties of the filaments in terms of curvature preference and persistence length as observed already for $\mathrm{Cdc1} 11_{\text {oct }}$ (Supplementary Figure S3) [13] and confirmed in Figure 4B.

The interaction with lipids favored the polymerization of $S h s 1_{\text {oct }}$ into filaments and the self-assembly of micrometric long filaments was enhanced (Figure 6, Supplementary Movies S2 and S3). As a comparison, in solution, Shs1 $1_{\text {oct }}$ never self-assemble into filaments longer than tens of nanometers.

On vesicles, Shs $1_{\text {oct }}$ assembled into a network of branched filaments (Figure 6B, upper right) or into sets of parallel and spaced filaments (Figure 6B, upper left). Sets of parallel filaments follow the contour of membranes and point towards the tip of deformed membranes, (Figure 6A, respectively, upper and bottom left). Filaments from branches extend from the edges of membranes at an angle of $10-60^{\circ}$ (Figure 6B). Finally, septins away from the edges of the membranes and cross-linking the vesicles formed the most disorganized structures.

After semi-automatic segmentation, filaments were indexed into three categories: (i) filaments in solution, (ii) filaments cross-linking vesicles or (iii) filaments displayed at the surface of the membranes. Only the third category was considered for henceforth statistical analysis. The length of the filaments varied from 80 to $870 \mathrm{~nm}(n$ $=255)$. Most were from 100 to $300 \mathrm{~nm}$ long $(66.7 \%$, Figure 6A), corresponding to 3-9 octamers. Each filament was $4 \pm 0.6 \mathrm{~nm}$ wide $(n=255)$, corresponding to one GBD of septins with two possible orientations on the surface of the vesicles. Within the same sheets, the distance between parallel filaments varied between 7 and $20 \mathrm{~nm}(11.5 \pm 3.4 \mathrm{~nm}, n=193)$. Densities displayed every $8.5-16.5 \mathrm{~nm}$ tethering the filaments were visible (Figure 6B, arrows), thus reminding Cdc11 paired filaments linked by Cdc3 and 12 CC domains [14].

\section{Synergistic role of nucleotides and lipids}

Since nucleotide binding and/or hydrolysis influence the high-ordered structures formed by septin proteins $[11,16,47-50]$, we decided to investigate the effect of either GDP or GTP on the polymerization of Shs1 $1_{\text {oct }}$ on a lipid monolayer as well.

To discriminate the role of nucleotide from the role of lipids, experiments were thus led both on a surface of a lipid monolayer and in solution. The protein complexes were expressed without adding nucleotides. A $40 \mu \mathrm{M}$ GDP was only present initially in the lysis buffer. The nucleotide turnover is slow but does take place as demonstrated in Khan et al. [11]. We assumed that nucleotide exchange was possible with a large excess of GTP or GTP $(4 \mathrm{mM})$. In solution and without or with nucleotides (GDP, GTP in excess, Shs $1_{\text {oct }}$ assembled into micrometric rings (Figure 7A, left panel (without excess nucleotides), and middle panel (with $4 \mathrm{mM}$ GTP)) or curved bundles (Figure 7A, right panel, $4 \mathrm{mM} \mathrm{GDP}$ ). The ultra-structural organization of Shs $1_{\text {oct }}$ is consequently unchanged in the presence of GTP or GDP nucleotides in excess, in solution. However, adding GTP- $\gamma$-S does not induce the assembly of shs1 complexes (Supplementary Figure S4) conversely to GTP and GDP. Consequently, the effect observed in the presence of GTP and GDP are promoted by an interaction between nucleotides and septin complexes rather than an electrostatic effect due to the high concentration of nucleotides. Cdc11 capped septin protomers polymerization is not perturbed by the presence of GTP- $\gamma$-S [51]. Nevertheless, structural studies showed differences when binding to different guanosine nucleotides, including analogs [52]. Cdc1 $1_{\text {oct }}$ is known to be more stable than $S h s 1_{\text {oct }}$, which is characterized by a lower amount of octameric complexes after purification (Supplementary Figure S1 [7]). The presence of GTP- $\gamma$-S could further disrupt the complex by inducing conformational changes.

On lipid monolayers, with an excess of GTP, Shs1 $1_{\text {oct }}$ assembled into novel bundles of filaments assembled into webs of 'starfish-like' structures with a central massive amount of filaments as a nucleation anchor for bundles of filaments (Figure 7B center, diameter of $112 \pm 26.5 \mathrm{~nm}(\min .66 .7 \mathrm{~nm}$; max. $197.6 \mathrm{~nm} ; n=36$ ) and Supplementary Figure S5). In excess of GDP, and interacting with a lipid monolayer, Shs $1_{\text {oct }}$ self-assembled into micron-scale sheets of densely packed interconnected bundles (Figure 7B, right panel). Their width varied between 18 and $64 \mathrm{~nm}(30.8 \pm 8.3 \mathrm{~nm}(n=69))$, corresponding to $4-16$ protomers. These observations were quantified and are recapitulated in Figure 7C. Consequently, the presence of nucleotides in excess combined with the presence of a lipid monolayer obviously induces the assembly of massive connected structures of filament bundles (Figure 7B, Supplementary Figure S5). Thus, a synergistic mechanism in between the lipid monolayer and nucleotide effect controls the oligomerization of Shs1 complexes.

\section{Modulation of Shs $1_{\text {oct }}$ self-assembly by binding nucleotides}

In GTPase proteins, residues involved in nucleotide binding and hydrolysis can (i) interact directly with the nucleotide or (ii) interact with the magnesium cations required for both the correct positioning of the nucleotide inside the active site and the hydrolysis of the phosphate $\gamma$ by GTPase proteins. A conserved serine found 

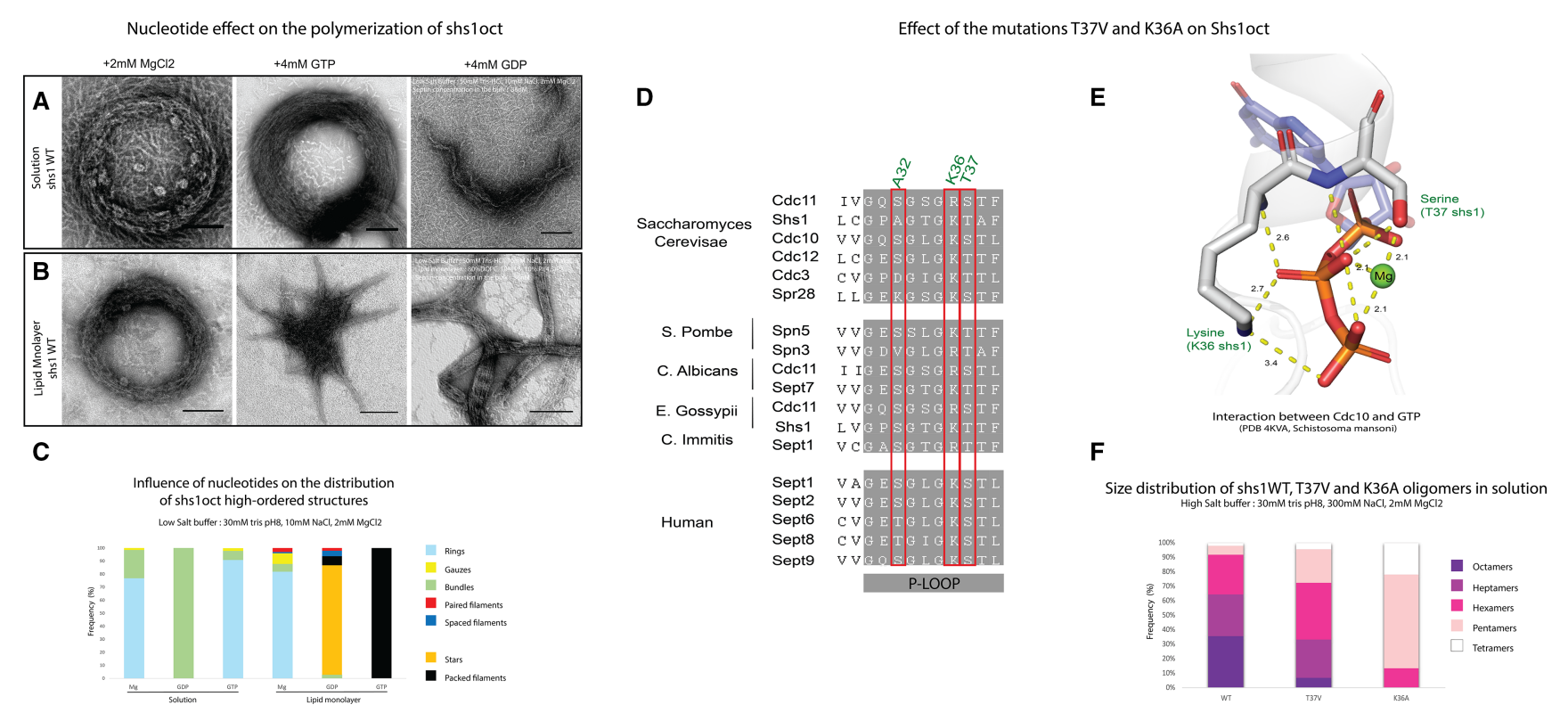

Figure 7. Role of nucleotides and lipids in the oligomerization of Shs1 ${ }_{\text {oct- }}$.

(A) Self-assembly of Shs $1_{\text {oct }}$ wild type in solution in the presence of $\mathrm{Mg}^{2+}$ alone (left), or with a $4 \mathrm{mM}$ excess of GTP (middle), and a 4 mM excess of GDP (right)). Scale bars: $100 \mathrm{~nm}$. (B) Self-assembly of Shs1 $1_{\text {oct }}$ wild type on the surface of a PI(4,5) $\mathrm{P}_{2}$ doped lipid monolayers in the presence of $\mathrm{Mg}^{2+}$ alone (left), or with a $4 \mathrm{mM}$ excess of GTP (middle), and a $4 \mathrm{mM}$ excess of GDP (right)). Scale bars: $100 \mathrm{~nm}$. (C) Graph displaying the relative distribution of the different ultra-structures obtained in solution (left) on lipid monolayers (right) with GTP or GDP or Magnesium. (D) Sequence alignment of the septin P-loop domain for different species. The residues A32 found in shs1 is either a threonin or a serine in other organisms. The residues K36 and T37, conserved, are also highlighted. (E) Structure (PDB code 4KVA) focusing on the GTP/GDP binding domain. The binding pocket is shown displaying the interactions between the involved residues (T37 and K36), the magnesium cation and the nucleotide. (F) Histograms describing the size distribution of purified Shs1 $1_{\text {oct }}$ protomers: Wild type (left), T37V (middle), K36A (right) including the mutation Thr37Val or Lys36Ala in Shs1 nucleotide-binding site is displayed in the chart at the bottom of the panel.

in Cdc11 is essential for the hydrolysis of GTP, but is not crucial for the interaction with GTP/GDP in both budding yeast and human [9,49]. Shs1 displays an alanine at this position (A32) (Figure 7D). This suggests that shs1 (deprived of this specific serine) might not be proficient to hydrolyze GTP even though the interaction with nucleotides is preserved. To understand the discrepancies observed for the polymerization of Shs $1_{\text {oct }}$ in the presence of different nucleotides, we focused our analysis on mutations that might perturb the interaction between Shs1 and GDP and/or GTP. More specifically we analyzed the role of the residues involved in the interaction with the phosphates $\beta$ and $\gamma$ of GTP.

The nucleotide-binding pocket from the septin proteins is highly conserved in sequence and structures, allowing to compare and align a large number of structures from the PDB, especially the structure 4KVA which is one of the rare GTP-bound septin structure available. A scheme representing an enlargement of this structure in the vicinity of the nucleotide-binding domain is displayed in Figure 7E.

By sequence alignment (Figure 7D) and structure analysis (see Method section, Figure 7E), Thr37 was found to interact directly with magnesium, buried in the G-interface between Cdc12 and Shs1 subunits. Lys36 is also an interesting candidate given it is a flexible residue potentially interacting directly with both hydroxyphosphates $\beta$ and $\gamma$ of GTP. The mutants Shs $1^{\text {Thr } 37 \mathrm{~V}}$ and Shs $1^{\text {Lys } 36 \mathrm{~A}}$ were thus designed, co-expressed with the other septins and purified as protomers (Shs $1^{\text {Thr37V }}$ oct and Shs $1^{\mathrm{Lys} 36 \mathrm{~A}}{ }_{\text {oct }}$ ). The different Shs1 mutants in the context of septin oligomers were analyzed by electron microscopy followed by 2D image analysis (Figure 7F).

Both mutants were strongly destabilized, demonstrating that nucleotide binding is crucial for the stability of the Shs $1_{\text {oct }}$ septin complex. Shs $1^{\mathrm{Thr} 37 \mathrm{~V}}{ }_{\text {oct }}$ generated only $6.6 \%$ of intact octamers, $26.4 \%$ of heptamers, $38.9 \%$ of hexameric complexes and more than $27 \%$ of shorter pentamers or even tetramers. The Shs $1_{\text {oct }}^{\text {Lys } 36 \mathrm{Ala}}$ complex was even more dramatically destabilized since the analysis demonstrated that only hexamers were recovered at best (14.5\%). Pentamers represented $69.5 \%$, tetramers and trimers represented, respectively, $23.7 \%$ and $2.3 \%$ of 
the septin complex distribution. This $2 \mathrm{D}$ analysis showed that barely any Shs1 subunit was stably incorporated within the complex. Indeed, from Finnigan et al. [53], Lys36 and Thr37 are localized within the p-loop of Shs1 which is crucial for the Shs1-Cdc12 interaction. This indeed explains why a low proportion of octamers is recovered. In solution and polymerizing conditions, Shs $1^{\mathrm{Thr} 37 \mathrm{~V}}$ oct assembled exclusively into individual short rods (not shown). The interaction of SHS1 with nucleotides is, therefore, required to ensure the stability of the Shs1 capped budding yeast septin octamer.

Taken together, our observations suggest that mutations affecting the binding of nucleotides directly to Shs1 monomer display a clear destabilization of septin oligomerization (Figure 7F). The effect of nucleotides on the organization of septins results at least from the coordination between Shs1 and GTP/GDP.

\section{Discussion}

Septins are multi-tasking proteins and ubiquitous in eukaryotes. Their versatility results from the capacity of septins to re-organize dynamically in vivo. To this end, the arrangement of septin subunits is modular. In budding yeast, the central hexameric septin palindromic core can indeed accommodate at both ends either Cdc11 or Shs1 $[5,7,11,16]$. The organization at nanometer resolution of the Shs1 octamer designated as Shs $1_{\text {oct }}$ had not been described before, bound to a biomimetic membrane. Hence, we have proposed here to analyze in details, using cell-free assays, how lipids and nucleotide binding can tune the ultra-structural organization of Shs $1_{\text {oct. }}$. To this end Shs1 was co-expressed with $\mathrm{Cdc} 3, \mathrm{Cdc10}$ and $\mathrm{Cdc1} 2$ to be considered in the context of an octamer. Indeed, in situ, the septins subunits co-localize with one another and are not relevant as single subunits.

A specific and significant role of nucleotides for septin polymerization is still unclear. However, the stability of Shs $1_{\text {oct }}$ depends on nucleotide binding. Indeed, we have shown here that point mutations (Lys36A and Thr37V affecting the nucleotide binding to Shs1) preclude the self-assembly of stable octamers (Figure 7). As shown previously [14], the stability of the Cdc1l complex is also sensitive to the coupling of septin subunits with GTP and GDP. The combination of a biomimetic membrane, together with GDP in excess, enhances the assembly of septin complexes and thus filaments by either facilitating the incorporation of free Shs1 subunits present in solution or by inducing filaments from hexameric Shs1 less septin complexes. However, adding GTP in excess is ineffective. In addition, wild type Shs $1_{\text {oct }}$ behave differently with GDP or GTP in excess on lipid monolayers. As shown by Weems and McMurray [16], throughout the complex assembly, the Shs1 subunit displays an enhanced affinity for Cdc12-GDP rather than to Cdc12-GTP. This behavior would explain a more efficient rescue of septin stability with an excess of GDP. Truncations within the long CTE of Shs1 alter the stability of Shs $1_{\text {oct }}$ as well, suggesting that the flexible CTE might be involved in inducing and stabilizing the Cdc12-Shs1 contact (Figure 5). To finely analyze our observations, coiled coil secondary structure predictions were performed using both MARCOIL and PCOILS. Both softwares agree on a coiled coil between residues 450 and 550. In addition, they detect, though with a lower probability and a lower redundancy between the softwares, an additional and distinct coiled coil between residues 375 and 445 . This secondary coiled coil would accommodate most of the so-called linker domain (residues 350-445). Hence this might explain why removing the linker domain and thereby the secondary coiled coil does not have the same impact as removing the whole CTE. Those two coiled coils might, therefore, not have the same role in assembling higher-ordered septin structures.

We have shown that $S h s 1_{\text {oct}}$, bound to lipids, can adopt a variety of ultra-structures recapitulated in Figure 8. These filamentous organizations observed in vitro are supported by prior observations of similar filamentous gauzes, rings and filaments carried out in situ [54,55]. In specific conditions (nucleotides in excess), we have observed massive bundled filaments arranged into star-shaped morphologies (Figure 7). Similarly, enhanced bundling was also observed by TIRF microscopy in the presence of nucleotides in excess [11]. Those ultrastructures might not be physiological but they reflect the plasticity of Shs $1_{\text {oct }}$ protomers. Some of the observed assemblies were already obtained without lipids (micrometric straight bundles or bundled rings, gauzes [7] while others seem to be mediated by membranes. On membranes, single or paired filaments $(100 \mathrm{~nm}$ long on monolayer, micrometers long on vesicles) are visualized while bundled filaments are exclusively assembled in solution. Thus Shs1 oct compete, in the presence of biomimetic membranes, between lateral interaction in between filaments and interaction with the membrane. Hence, in situ, Shs $1_{\text {oct }}$, in the vicinity of the plasma membrane might preferentially associate into networks of filaments rather than into aggregated bundles. Besides, peculiar 'wheel-like' organizations, accommodating two $S h s 1_{\text {oct }}$, have been highlighted. Even though those peculiar assemblies are clearly visible in the figures of published reports $[7,42]$, they had not been 


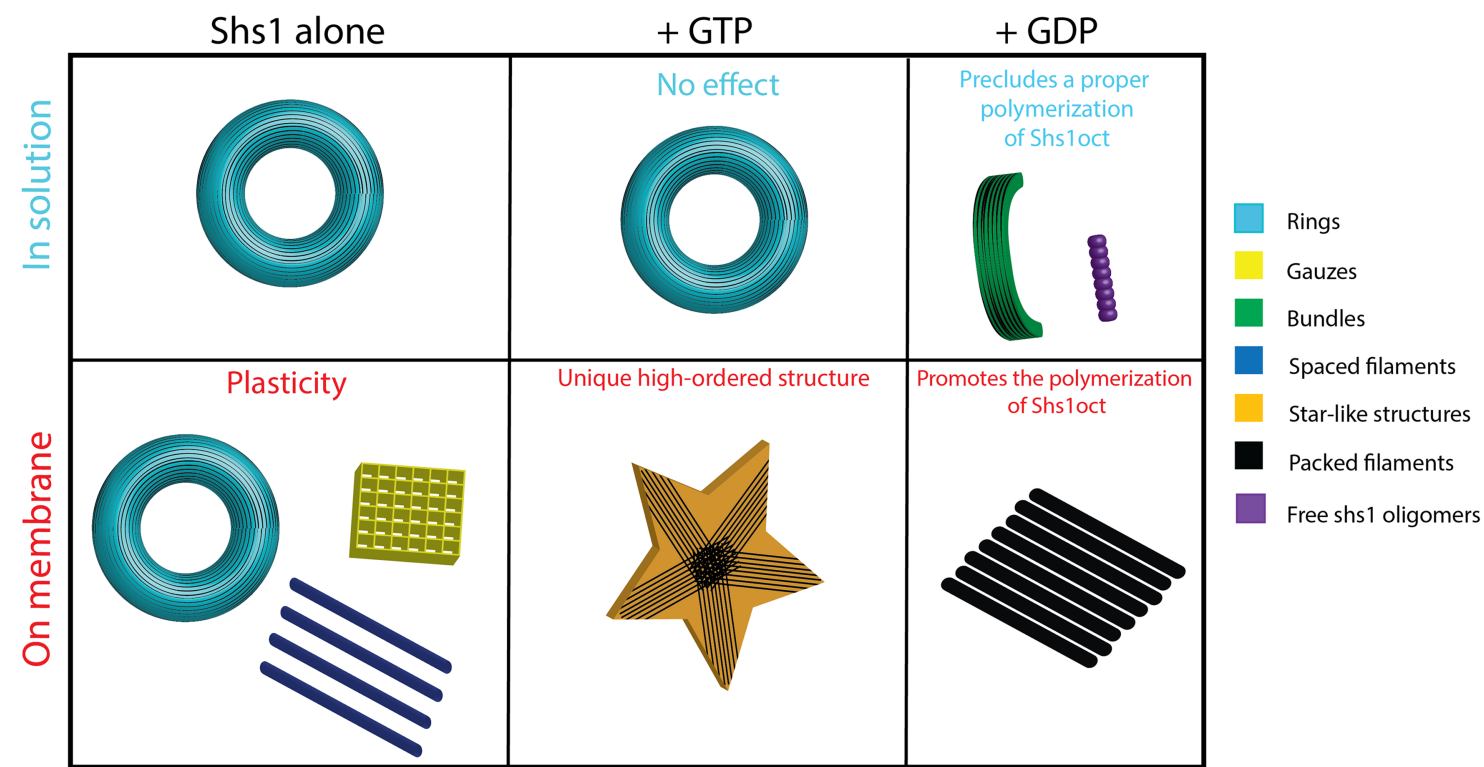

Figure 8. Synergetic role of lipids and nucleotides on the assembly of Shs1 oct: a schematic representation.

Variety of ultra-structures obtained in solution (top row) or on biomimetic membranes (bottom row) in an excess of GDP (right) or GTP (middle). Similar color codes are used in Figures 3 and 7.

commented. In vivo, those 'wheels' might stabilize septin protomers and thus prevent any aggregation of freestanding cytoplasmic complexes and build up a pool of soluble Shs1 capped septin protomer. Indeed, in the course of the cell cycle, septins undergo major re-arrangements and cycles of assembly/disassembly. Hence, upon disassembly, soluble wheel-like assemblies might be preferable than aggregated bundles.

As opposed to Cdc1 $1_{\text {oct }}$, Shs $1_{\text {oct }}$ does not exclusively bind to $\mathrm{PI}(4,5) \mathrm{P}_{2}$. Indeed, Shsloct self-organize similarly on lipid monolayers doped with $\mathrm{PIP}, \mathrm{PI}(4,5) \mathrm{P}_{2}$ or $\mathrm{PI}(3,4,5) \mathrm{P}_{3}$. To analyze in details possible molecular cues that might account for the discrepancies between $S h s 1_{\text {oct }}$ and $C d c 11_{\text {oct }}$ we have undertaken molecular modeling assays and showed how specific and fine residue positioning could be responsible for these differences.

\section{Conclusion}

Septin proteins display highly dynamic structures since they undergo major remodeling events in situ, for instance throughout cell division. This plasticity is certainly regulated, in vivo, by multiple factors. It is thus necessary to dissect their specific role to understand how septins are regulated. Following this strategy, we chose to isolate Shs1 complexes in vitro and have demonstrated that a combination of factors (nucleotides, lipids, ionic strength) are responsible for the plasticity of those septin protomers. They control and regulate the self-assembly of the mitotic septin from S. cerevisiae, alone or in synergy. We believe that the biomimetic environment used in this study thereby reflects, at least partly, the role and behavior of septins within a cellular environment.

\section{Competing Interests}

The authors declare that there are no competing interests associated with the manuscript.

\section{Funding}

CNRS, Agence Nationale pour la Recherche: grants ANR-13-JSV8-0002-01 and ANR-17-CE13-0014 to A.B; ANR10-INBS-04; Equipment d'excellence CACSICE.

\section{Author Contributions}

C.T. and A.B. designed research. C.T. and A.B. expressed and purified the septin complexes. C.T., A.B., G.P.-A. and A.D.C. performed the cryo-electron microscopy experiments. R.B. and C.T. performed the segmentation of 
the cryo-tomogram and data analysis. C.T. performed modelization and docking experiments. The results and their interpretation were discussed by all of the authors. C.T. and A.B. wrote the manuscript.

\section{Acknowledgements}

This work was supported by the Institut Curie, Centre National de la Recherche Scientifique (CNRS), the Agence Nationale pour la Recherche (grant ANR-13-JSV8-0002-01 and ANR-17-CE13-0014 to A.B). We thank the Cell and Tissue Imaging (PICT-IBiSA), Institut Curie, member of the French National Research Infrastructure France-Biolmaging (ANR10-INBS-04). We thank Fahima Faqir for the cloning of certain mutant. We finally thank Dr. Eva Nogales for kindly providing Shs1 plasmid. We thank the platform UTechS UBI at Institut Pasteur (Paris) and M. Nilges and the Equipment d'excellence CACSICE for providing the Falcon II direct electron detector.

\section{Abbreviations}

CC, coiled-coil domain; CTE, C-terminal extension; GBD, guanine nucleotide-binding domain.

\section{References}

1 Wloka, C. and Bi, E. (2012) Mechanisms of cytokinesis in budding yeast. Cytoskeleton (Hoboken) 69, 710-726 https://doi.org/10.1002/cm.21046

2 Roncero, C. and Sanchez, Y. (2010) Cell separation and the maintenance of cell integrity during cytokinesis in yeast: the assembly of a septum. Yeast 27, 521-530 https://doi.org/10.1002/yea.1779

3 Glomb, 0. and Gronemeyer, T. (2016) Septin organization and functions in budding yeast. Front. Cell Dev. Biol. 4, 123 https://doi.org/10.3389/fcell. 2016.00123

4 Oh, Y. and Bi, E. (2011) Septin structure and function in yeast and beyond. Trends Cell Biol. 21, 141-148 https://doi.org/10.1016/j.tcb.2010.11.006

5 Bertin, A., McMurray, M.A., Grob, P., Park, S.S., Garcia, III, G., Patanwala, I. et al. (2008) Saccharomyces cerevisiae septins: supramolecular organization of heterooligomers and the mechanism of filament assembly. Proc. Natl. Acad. Sci. U.S.A. 105, 8274-8279 https://doi.org/10.1073/pnas. 0803330105

6 Finnigan, G.C., Duvalyan, A., Liao, E.N., Sargsyan, A. and Thorner, J. (2016) Detection of protein-protein interactions at the septin collar in Saccharomyces cerevisiae using a tripartite split-GFP system. Mol. Biol. Cell 27, 2708-2725 https://doi.org/10.1091/mbc.e16-05-0337

7 Garcia, III, G., Bertin, A., Li, Z., Song, Y., McMurray, M.A., Thorner, J. et al. (2011) Subunit-dependent modulation of septin assembly: budding yeast septin Shs1 promotes ring and gauze formation. J. Cell Biol. 195, 993-1004 https://doi.org/10.1083/jcb.201107123

8 McMurray, M.A., Bertin, A., Garcia, III, G., Lam, L., Nogales, E. and Thorner, J. (2011) Septin filament formation is essential in budding yeast. Dev. Cell 20, 540-549 https://doi.org/10.1016/j.devcel.2011.02.004

9 Versele, M. and Thorner, J. (2004) Septin collar formation in budding yeast requires GTP binding and direct phosphorylation by the PAK, Cla4. J. Cell Biol. 164, 701-715 https://doi.org/10.1083/jcb.200312070

10 Weirich, C.S., Erzberger, J.P. and Barral, Y. (2008) The septin family of GTPases: architecture and dynamics. Nat. Rev. Mol. Cell Biol. 9, 478-489 https://doi.org/10.1038/nrm2407

11 Khan, A., Newby, J. and Gladfelter, A.S. (2018) Control of septin filament flexibility and bundling by subunit composition and nucleotide interactions. Mol. Biol. Cell 29, 702-712 https://doi.org/10.1091/mbc.E17-10-0608

12 Beber, A., Alqabandi, M., Prevost, C., Viars, F., Levy, D., Bassereau, P. et al. (2018) Septin-based readout of PI(4,5)P2 incorporation into membranes of giant unilamellar vesicles. Cytoskeleton (Hoboken) 76, 92-103 https://doi.org/10.1002/cm.21480

13 Beber, A., Taveneau, C., Nania, M., Tsai, F.C., Di Cicco, A., Bassereau, P. et al. (2019) Membrane reshaping by micrometric curvature sensitive septin filaments. Nat. Commun. 10, 420 https://doi.org/10.1038/s41467-019-08344-5

14 Bertin, A., McMurray, M.A., Thai, L., Garcia, III, G., Votin, V., Grob, P. et al. (2010) Phosphatidylinositol-4,5-bisphosphate promotes budding yeast septin filament assembly and organization. J. Mol. Biol. 404, 711-731 https://doi.org/10.1016/j.jmb.2010.10.002

15 Booth, E.A., Vane, E.W., Dovala, D. and Thorner, J. (2015) A forster resonance energy transfer (FRET)-based system provides insight into the ordered assembly of yeast septin hetero-octamers. J. Biol. Chem. 290, 28388-28401 https://doi.org/10.1074/jbc.M115.683128

16 Weems, A. and McMurray, M. (2017) The step-wise pathway of septin hetero-octamer assembly in budding yeast. elife 6, e23689 https://doi.org/10. 7554/eLife.23689

17 Arganda-Carreras, I., Kaynig, V., Rueden, C., Eliceiri, K.W., Schindelin, J., Cardona, A. et al. (2017) Trainable Weka segmentation: a machine learning tool for microscopy pixel classification. Bioinformatics 33, 2424-2426 https://doi.org/10.1093/bioinformatics/btx180

18 Kremer, J.R., Mastronarde, D.N. and McIntosh, J.R. (1996) Computer visualization of three-dimensional image data using IMOD. J. Struct. Biol. 116, 71-76 https://doi.org/10.1006/jsbi.1996.0013

19 Rigort, A., Gunther, D., Hegerl, R., Baum, D., Weber, B., Prohaska, S. et al. (2012) Automated segmentation of electron tomograms for a quantitative description of actin filament networks. J. Struct. Biol. 177, 135-144 https://doi.org/10.1016/j.jsb.2011.08.012

20 Descoteaux, M., Audette, M., Chinzei, K. and Siddiqi, K. (2006) Bone enhancement filtering: application to sinus bone segmentation and simulation of pituitary surgery. Comput. Aided Surg. 11, 247-255 https://doi.org/10.3109/10929080601017212

21 Tang, G., Peng, L., Baldwin, P.R., Mann, D.S., Jiang, W., Rees, I. et al. (2007) EMAN2: an extensible image processing suite for electron microscopy. J. Struct. Biol. 157, 38-46 https://doi.org/10.1016/j.jsb.2006.05.009

22 Frank, J., Radermacher, M., Penczek, P., Zhu, J., Li, Y., Ladjadj, M. et al. (1996) SPIDER and WEB: processing and visualization of images in 3D electron microscopy and related fields. J. Struct. Biol. 116, 190-199 https://doi.org/10.1006/jsbi.1996.0030

23 Armougom, F., Moretti, S., Poirot, O., Audic, S., Dumas, P., Schaeli, B. et al. (2006) Expresso: automatic incorporation of structural information in multiple sequence alignments using 3D-Coffee. Nucleic Acids Res. 34, W604-W608 https://doi.org/10.1093/nar/gkl092

24 Biegert, A., Mayer, C., Remmert, M., Soding, J. and Lupas, A.N. (2006) The MPI bioinformatics toolkit for protein sequence analysis. Nucleic Acids Res. 34, W335-W339 https://doi.org/10.1093/nar/gkl217 
25 Jones, D.T. (1999) Protein secondary structure prediction based on position-specific scoring matrices. J. Mol. Biol. 292, 195-202 https://doi.org/10. 1006/jmbi.1999.3091

26 Heffernan, R., Yang, Y., Paliwal, K. and Zhou, Y. (2017) Capturing non-local interactions by long short-term memory bidirectional recurrent neural networks for improving prediction of protein secondary structure, backbone angles, contact numbers and solvent accessibility. Bioinformatics 33, 2842-2849 https://doi.org/10.1093/bioinformatics/btx218

27 Yan, R., Xu, D., Yang, J., Walker, S. and Zhang, Y. (2013) A comparative assessment and analysis of 20 representative sequence alignment methods for protein structure prediction. Sci. Rep. 3, 2619 https://doi.org/10.1038/srep02619

28 Wang, S., Peng, J., Ma, J. and Xu, J. (2016) Protein secondary structure prediction using deep convolutional neural fields. Sci. Rep. 6, 18962 https://doi.org/10.1038/srep18962

29 Klausen, M.S., Jespersen, M.C., Nielsen, H., Jensen, K.K., Jurtz, V.I., Sonderby, C.K. et al. (2019) NetSurfP-2.0: improved prediction of protein structural features by integrated deep learning. Proteins 87, 520-527 https://doi.org/10.1002/prot.25674

30 Kall, L., Krogh, A. and Sonnhammer, E.L. (2005) An HMM posterior decoder for sequence feature prediction that includes homology information. Bioinformatics 21 Suppl 1, i251-i257 https://doi.org/10.1093/bioinformatics/bti1014

31 Jones, D.T. and Cozzetto, D. (2015) DISOPRED3: precise disordered region predictions with annotated protein-binding activity. Bioinformatics 31, 857-863 https://doi.org/10.1093/bioinformatics/btu744

32 Hanson, J., Yang, Y., Paliwal, K. and Zhou, Y. (2017) Improving protein disorder prediction by deep bidirectional long short-term memory recurrent neural networks. Bioinformatics 33, 685-692 https://doi.org/10.1093/bioinformatics/btw678

33 Dosztanyi, Z., Csizmok, V., Tompa, P. and Simon, I. (2005) The pairwise energy content estimated from amino acid composition discriminates between folded and intrinsically unstructured proteins. J. Mol. Biol. 347, 827-839 https://doi.org/10.1016/j.jmb.2005.01.071

34 Lupas, A., Van Dyke, M. and Stock, J. (1991) Predicting coiled coils from protein sequences. Science 252, 1162-1164 https://doi.org/10.1126/ science.252.5009.1162

35 Gruber, M., Soding, J. and Lupas, A.N. (2006) Comparative analysis of coiled-coil prediction methods. J. Struct. Biol. 155, 140-145 https://doi.org/10 1016/j.jsb.2006.03.009

36 Delorenzi, M. and Speed, T. (2002) An HMM model for coiled-coil domains and a comparison with PSSM-based predictions. Bioinformatics 18, 617-625 https://doi.org/10.1093/bioinformatics/18.4.617

37 Zimmermann, L., Stephens, A., Nam, S.Z., Rau, D., Kubler, J., Lozajic, M. et al. (2018) A completely reimplemented MPI bioinformatics toolkit with a new HHpred server at its core. J. Mol. Biol. 430, 2237-2243 https://doi.org/10.1016/j.jmb.2017.12.007

38 Trigg, J., Gutwin, K., Keating, A.E. and Berger, B. (2011) Multicoil2: predicting coiled coils and their oligomerization states from sequence in the twilight zone. PLoS One 6, e23519 https://doi.org/10.1371/journal.pone.0023519

39 Zhang, Y. (2008) I-TASSER server for protein 3D structure prediction. BMC Bioinform. 9, 40 https://doi.org/10.1186/1471-2105-9-40

40 Brausemann, A., Gerhardt, S., Schott, A.K., Einsle, O., Grosse-Berkenbusch, A., Johnsson, N. et al. (2016) Crystal structure of Cdc11, a septin subunit from Saccharomyces cerevisiae. J. Struct. Biol. 193, 157-161 https://doi.org/10.1016/j.jsb.2016.01.004

41 Grosdidier, A., Zoete, V. and Michielin, 0. (2011) Swissdock, a protein-small molecule docking web service based on EADock DSS. Nucleic Acids Res. 39, W270-W277 https://doi.org/10.1093/nar/gkr366

42 Kinoshita, M., Field, C.M., Coughlin, M.L., Straight, A.F. and Mitchison, T.J. (2002) Self- and actin-templated assembly of Mammalian septins. Dev. Cell 3, 791-802 https://doi.org/10.1016/S1534-5807(02)00366-0

43 Tanaka-Takiguchi, Y., Kinoshita, M. and Takiguchi, K. (2009) Septin-mediated uniform bracing of phospholipid membranes. Curr. Biol. 19, 140-145 https://doi.org/10.1016/j.cub.2008.12.030

44 Zhang, J., Kong, C., Xie, H., McPherson, P.S., Grinstein, S. and Trimble, W.S. (1999) Phosphatidylinositol polyphosphate binding to the mammalian septin H5 is modulated by GTP. Curr. Biol. 9, 1458-1467 https://doi.org/10.1016/S0960-9822(00)80115-3

45 Tokmakov, A.A., Kurotani, A., Takagi, T., Toyama, M., Shirouzu, M., Fukami, Y. et al. (2012) Multiple post-translational modifications affect heterologous protein synthesis. J. Biol. Chem. 287, 27106-27116 https://doi.org/10.1074/jbc.M112.366351

46 Cannon, K.S., Woods, B.L., Crutchley, J.M. and Gladfelter, A.S. (2019) An amphipathic helix enables septins to sense micrometer-scale membrane curvature. J. Cell Biol. 218, 1128-1137 https://doi.org/10.1083/jcb.201807211

47 Johnson, E., Seiradake, E., Jones, E.Y., Davis, I., Grunewald, K. and Kaufmann, R. (2015) Correlative in-resin super-resolution and electron microscopy using standard fluorescent proteins. Sci. Rep. 5, $9583 \mathrm{https://doi.org/10.1038/srep09583}$

48 Nagaraj, S., Rajendran, A., Jackson, C.E. and Longtine, M.S. (2008) Role of nucleotide binding in septin-septin interactions and septin localization in Saccharomyces cerevisiae. Mol. Cell. Biol. 28, 5120-5137 https://doi.org/10.1128/MCB.00786-08

49 Sirajuddin, M., Farkasovsky, M., Zent, E. and Wittinghofer, A. (2009) GTP-induced conformational changes in septins and implications for function. Proc. Natl. Acad. Sci. U.S.A. 106, 16592-16597 https://doi.org/10.1073/pnas.0902858106

50 Zent, E. and Wittinghofer, A. (2014) Human septin isoforms and the GDP-GTP cycle. Biol. Chem. 395, 169-180 https://doi.org/10.1515/hsz-2013-0268

51 Mendoza, M., Hyman, A.A. and Glotzer, M. (2002) GTP binding induces filament assembly of a recombinant septin. Curr. Biol. 12, 1858-1863 https://doi.org/10.1016/S0960-9822(02)01258-7

52 Castro, D., da Silva, S.M.O., Pereira, H.D., Macedo, J.N.A., Leonardo, D.A., Valadares, N.F. et al. (2020) A complete compendium of crystal structures for the human SEPT3 subgroup reveals functional plasticity at a specific septin interface. IUCrJ 7, 462-479 https://doi.org/10.1107/ S2052252520002973

53 Finnigan, G.C., Takagi, J., Cho, C. and Thorner, J. (2015) Comprehensive genetic analysis of paralogous terminal septin subunits Shs1 and Cdc11 in Saccharomyces cerevisiae. Genetics 200, 821-841 https://doi.org/10.1534/genetics.115.176495

54 Bertin, A., McMurray, M.A., Pierson, J., Thai, L., McDonald, K.L., Zehr, E.A. et al. (2012) Three-dimensional ultrastructure of the septin filament network in Saccharomyces cerevisiae. Mol. Biol. Cell 23, 423-432 https://doi.org/10.1091/mbc.e11-10-0850

55 Ong, K., Wloka, C., Okada, S., Svitkina, T. and Bi, E. (2014) Architecture and dynamic remodelling of the septin cytoskeleton during the cell cycle. Nat. Commun. 5, 5698 https://doi.org/10.1038/ncomms6698

56 Vrabioiu, A.M. and Mitchison, T.J. (2006) Structural insights into yeast septin organization from polarized fluorescence microscopy. Nature $\mathbf{4 4 3}$ 466-469 https://doi.org/10.1038/nature05109 